\title{
Article \\ Role of Seasonality and Fire in Regulating the Enzymatic Activities in Soils Covered by Different Vegetation in a Mediterranean Area
}

\author{
Valeria Memoli ${ }^{1}$, Lucia Santorufo ${ }^{1,2, *(\mathbb{D})}$, Giorgia Santini ${ }^{1}{ }^{(D}$, Paola Musella ${ }^{1}$, Rossella Barile ${ }^{3}$, \\ Anna De Marco ${ }^{2,4}\left(\mathbb{D}\right.$, Gabriella Di Natale ${ }^{5,6} \mathbb{D}$, Marco Trifuoggi ${ }^{5}$ and Giulia Maisto ${ }^{1,2}$
}

check for updates

Citation: Memoli, V.; Santorufo, L.; Santini, G.; Musella, P.; Barile, R.; De Marco, A.; Di Natale, G.; Trifuoggi, M.; Maisto, G. Role of Seasonality and Fire in Regulating the Enzymatic Activities in Soils Covered by Different Vegetation in a Mediterranean Area. Appl. Sci. 2021, 11, 8342. https://doi.org/10.3390/ app11188342

Academic Editor: Daniel Dias

Received: 28 July 2021

Accepted: 6 September 2021

Published: 8 September 2021

Publisher's Note: MDPI stays neutral with regard to jurisdictional claims in published maps and institutional affiliations.

Copyright: (c) 2021 by the authors. Licensee MDPI, Basel, Switzerland. This article is an open access article distributed under the terms and conditions of the Creative Commons Attribution (CC BY) license (https:/ / creativecommons.org/licenses/by/ $4.0 /)$.
1 Dipartimento di Biologia, Università degli Studi di Napoli Federico II, Via Cinthia, 80126 Napoli, NA, Italy; valeria.memoli@unina.it (V.M.); giorgia.santini@unina.it (G.S.); paola.musella@uclouvain.be (P.M.); g.maisto@unina.it (G.M.)

2 BAT Center-Interuniversity Center for Studies on Bioinspired Agro-Environmental Technology, University of Naples Federico II, 80126 Napoli, NA, Italy; ademarco@unina.it

3 Parco Nazionale del Vesuvio, Via Palazzo del Principe c/o Castello Mediceo, 80044 Ottaviano, NA, Italy; rbarile@epnv.it

4 Dipartimento di Farmacia, Università degli Studi di Napoli Federico II, Via Montesano, 80131 Napoli, NA, Italy

5 Dipartimento di Scienze Chimiche, Università degli Studi di Napoli Federico II, Via Cinthia, 80126 Napoli, NA, Italy; gabriella.dinatale@unina.it (G.D.N.); marco.trifuoggi@unina.it (M.T.)

6 CeSMA-Centro Servizi Metrologici e Tecnologici Avanzati, Università degli Studi di Napoli Federico II, Corso Nicolangelo Protopisani, 80146 San Giovanni a Teduccio, NA, Italy

* Correspondence: lucia.santorufo@unina.it

Abstract: As they quickly respond to environmental conditions, soil enzymes, involved in nutrient cycles, are considered good indicators of soil quality. The Mediterranean area is a peculiar environment for climatic conditions and for fire frequency. Therefore, the research aimed to evaluate the role of seasonality and fire on enzymatic activities (i.e., hydrolase, dehydrogenase, and $\beta$-glucosidase) in soils covered by herbs, black locust, pine, and holm oak. In addition, the main soil abiotic properties that drive the enzymatic activities were also investigated. In order to achieve the aims, surface soils were collected in unburnt and burnt areas and characterized for water and organic matter contents, $\mathrm{pH}$, concentrations of $\mathrm{C}$ and $\mathrm{N}$, and available fractions of $\mathrm{Al}, \mathrm{Ca}, \mathrm{Cu}, \mathrm{Fe}, \mathrm{Mg}, \mathrm{Mn}, \mathrm{Na}$, and $\mathrm{Pb}$. The results highlighted that the soil enzymatic activities were mainly affected by seasonality more than by fire; in unburnt soils, their main drivers were nutrient availabilities, whereas, in burnt soils they were $\mathrm{pH}$, water and organic matter contents, $\mathrm{C}$ and $\mathrm{N}$ concentrations, and both nutrient and metal availabilities. Finally, holm oak, as compared with herbs, pine, and black locust, conferred higher stability to soils that were affected by seasonality and fire.

Keywords: hydrolase; dehydrogenase; $\beta$-glucosidase; nutrient availability; metal availability

\section{Introduction}

Soil enzymes, playing a fundamental role in organic matter decomposition, are involved in nutrient cycling and are critical to soil functioning [1]. As they quickly respond to environmental conditions, they are also considered good indicators of soil quality [2].

Their spatial and temporal trends are strongly regulated by soil microorganisms, but also by soil properties [2,3]. Seasonality, responsible for changing in temperature, humidity, and precipitation, directly and indirectly alters soil enzymatic activity [4,5]. Seasonal fluctuations in soil enzymatic activity are reported for several ecosystems such as tropical [6], temperate [7], and alpine forests [8]. Moreover, it is well documented that temperature and precipitation are the main drivers of soil enzymatic activity $[9,10]$.

Besides, soil properties such as $\mathrm{pH}, \mathrm{C} / \mathrm{N}$ ratio, and element content also play a fundamental role in regulating the soil enzymatic activity [11,12]. However, there is a lack 
of basic information regarding whether the critical drivers of seasonal soil enzyme activity differ among vegetation types [5].

In Mediterranean regions, fires are among the main disturbance factors that cause deep changes in soil abiotic and biotic properties and alter soil quality [13-16]. Fire effects on soil enzymatic activities are direct as they cause protein denaturation, temporary soil sterilization, and nutrient mineralization $[17,18]$, and indirect as they cause changes in soil microbial and plant communities [19-22].

Among the soil enzymatic activities, hydrolase, dehydrogenase, and $\beta$-glucosidase are good indicators of soil microbial activity $[23,24]$. In fact, hydrolase, intermediating hydrolytic reactions, allows for the estimation of total soil microbial activity $[15,25]$. In addition, extracellular hydrolytic enzyme activities (dehydrogenase and $\beta$-glucosidase) are strongly affected by fire, as reported by several authors in studied performed in boreal and temperate forests $[2,26,27]$. Dehydrogenase, oxidizing the soil organic matter by transferring protons and electrons from substrates to acceptors, is a well-known biological indicator of overall microbial respiratory activity [28]. $\beta$-glucosidase is involved in C mineralization and provides information on the degradation of cellulose, which is the most common polysaccharide in nature [29].

Investigations about the relationships between temporal trend of soil enzymatic activities and soil properties can provide useful information not only for understanding the soil processes, but also for predicting their responses to environmental change [5]. As research focusing on the role of vegetation cover upon the soil enzymatic activities in areas affected by fire is scarce, the research aimed: (i) to evaluate the seasonal trends of enzymatic activities in unburnt and burnt soils covered by the same vegetation cover; (ii) to compare the role of each vegetation cover on soil enzymatic activities in unburnt and burnt soils; and (iii) to highlight, among the investigated abiotic soil properties, the main drivers of the soil enzymatic activities in unburnt and burnt areas. In order to achieve the aims, surface soils were collected under herbs, black locust, pine, and holm oak in unburnt and burnt soils inside the Vesuvius National Park located in the Mediterranean area of Southern Italy. The collected soils were characterized for the main abiotic soil properties (i.e., water and organic matter contents, $\mathrm{pH}$, concentrations of $\mathrm{C}$ and $\mathrm{N}$, and available fractions of $\mathrm{Al}, \mathrm{Ca}$, $\mathrm{Cu}, \mathrm{Fe}, \mathrm{Mg}, \mathrm{Mn}, \mathrm{Na}$ and $\mathrm{Pb}$ ) and enzymatic activities (i.e., hydrolase, dehydrogenase, and $\beta$-glucosidase). The findings provide useful information about the integrated effects of seasons, vegetation cover, and fire occurrence on soil abiotic properties that, in turn, can drive the soil enzymatic activities.

\section{Materials and Methods}

\subsection{Study Area and Soil Sampling}

The research was performed in the Vesuvius National Park located in the surrounding of Naples, Southern Italy. The vegetation inside the National Park were represented by holm oaks (Quercus ilex L.), pines (Pinus nigra L.) and shrubs and herbs (such as Myrtus communis L., Laurus nobilis L., Viburnum tinus L., Cistus sp., Ginesta sp.) [14]. Additionally, a few patches of black locust (Robinia pseudoacacia L.) were present in some areas of the park. In summer 2017, the investigated area was affected by a fire of level four on the soil burn severity index [30], that lasted three days (11th, 12th, and 16th of July), and that reached a temperature which completely consumed the forest floor [31].

The soil sampling was carried out every three months from summer 2018 to spring 2019 within two consecutive days and after seven days without rainfall at six sites (Table 1) covered by herbs $(\mathrm{H})$, by black locust $(\mathrm{BL})$, by pine $(\mathrm{P})$, and by holm oak $(\mathrm{HO})$ and that represent the main vegetation kinds of the Vesuvius National Park [15,16]. For each vegetation cover, three sites were chosen in the burnt area (B) and three in the unburnt (UB) area (Table 1), located in proximity of the burnt one, for a total of twenty-four sites (three sites in the burnt area and three sites in the unburnt area covered by the four vegetation types) for each season. Eight cores of surface (depth: 0-10 cm) soils were collected at each of the twenty-four sites after removal of ash or litter, respectively, at burnt and unburnt 
sites, and mixed together in order to obtain a homogeneous sample. In order to avoid disturbances for the microbial biomass, the soil samples were transported on ice to the laboratory [32].

Table 1. Sampling sites collected in the investigated area. Site description of the unburnt and burnt soils sampled inside the Vesuvius National Park under different vegetation covers.

\begin{tabular}{|c|c|c|c|}
\hline Sampling Site & Vegetation cover & Fire & Coordinates \\
\hline HO1 & Holm Oak & Burnt & $\begin{array}{l}40^{\circ} 80^{\prime} 72^{\prime \prime} \\
14^{\circ} 43^{\prime} 46^{\prime \prime}\end{array}$ \\
\hline $\mathrm{HO} 2$ & Holm Oak & Unburnt & $\begin{array}{l}40^{\circ} 80^{\prime} 88^{\prime \prime} \\
14^{\circ} 43^{\prime} 92^{\prime \prime}\end{array}$ \\
\hline $\mathrm{HO} 3$ & Holm Oak & Burnt & $\begin{array}{l}40^{\circ} 80^{\prime} 23^{\prime \prime} \\
14^{\circ} 44^{\prime} 15^{\prime \prime}\end{array}$ \\
\hline $\mathrm{HO} 4$ & Holm Oak & Unburnt & $\begin{array}{l}40^{\circ} 81^{\prime} 03^{\prime \prime} \\
14^{\circ} 40^{\prime} 86^{\prime \prime}\end{array}$ \\
\hline HO5 & Holm Oak & Burnt & $\begin{array}{l}40^{\circ} 81^{\prime} 67^{\prime \prime} \\
14^{\circ} 40^{\prime} 86^{\prime \prime}\end{array}$ \\
\hline HO6 & Holm Oak & Unburnt & $\begin{array}{l}40^{\circ} 81^{\prime} 99^{\prime \prime} \\
14^{\circ} 39^{\prime} 96^{\prime \prime}\end{array}$ \\
\hline P1 & Pine & Burnt & $\begin{array}{l}40^{\circ} 79^{\prime} 71^{\prime \prime} \\
14^{\circ} 43^{\prime} 87^{\prime \prime}\end{array}$ \\
\hline P2 & Pine & Unburnt & $\begin{array}{l}40^{\circ} 80^{\prime} 19^{\prime \prime} \\
14^{\circ} 43^{\prime} 85^{\prime \prime}\end{array}$ \\
\hline P3 & Pine & Burnt & $\begin{array}{l}40^{\circ} 80^{\prime} 19^{\prime \prime} \\
14^{\circ} 26^{\prime} 13^{\prime \prime}\end{array}$ \\
\hline P4 & Pine & Burnt & $\begin{array}{l}40^{\circ} 81^{\prime} 31^{\prime \prime} \\
14^{\circ} 43^{\prime} 80^{\prime \prime}\end{array}$ \\
\hline P5 & Pine & Unburnt & $\begin{array}{l}40^{\circ} 83^{\prime} 10^{\prime \prime} \\
14^{\circ} 25^{\prime} 02^{\prime \prime}\end{array}$ \\
\hline P6 & Pine & Unburnt & $\begin{array}{l}40^{\circ} 82^{\prime} 41^{\prime \prime} \\
14^{\circ} 39^{\prime} 18^{\prime \prime}\end{array}$ \\
\hline BL1 & Black Locust & Unburnt & $\begin{array}{l}40^{\circ} 81^{\prime} 20^{\prime \prime} \\
14^{\circ} 44^{\prime} 07^{\prime \prime}\end{array}$ \\
\hline BL2 & Black Locust & Burnt & $\begin{array}{l}40^{\circ} 80^{\prime} 88^{\prime \prime} \\
14^{\circ} 43^{\prime} 92^{\prime \prime}\end{array}$ \\
\hline BL3 & Black Locust & Unburnt & $\begin{array}{c}40^{\circ} 82^{\prime} 26^{\prime \prime} \\
14^{\circ} 43^{\prime} 55\end{array}$ \\
\hline BL4 & Black Locust & Burnt & $\begin{array}{l}40^{\circ} 82^{\prime} 86^{\prime \prime} \\
14^{\circ} 43^{\prime} 04^{\prime \prime}\end{array}$ \\
\hline BL5 & Black Locust & Burnt & $\begin{array}{l}40^{\circ} 82^{\prime} 36^{\prime \prime} \\
14^{\circ} 43^{\prime} 53^{\prime \prime}\end{array}$ \\
\hline BL6 & Black Locust & Unburnt & $\begin{array}{l}40^{\circ} 82^{\prime} 13^{\prime \prime} \\
14^{\circ} 43^{\prime} 62^{\prime \prime}\end{array}$ \\
\hline $\mathrm{H} 1$ & Herbs & Burnt & $\begin{array}{l}40^{\circ} 81^{\prime} 31^{\prime \prime} \\
14^{\circ} 43^{\prime} 66^{\prime \prime}\end{array}$ \\
\hline $\mathrm{H} 2$ & Herbs & Unburnt & $\begin{array}{l}40^{\circ} 82^{\prime} 30^{\prime \prime} \\
14^{\circ} 39^{\prime} 96^{\prime \prime}\end{array}$ \\
\hline $\mathrm{H} 3$ & Herbs & Burnt & $\begin{array}{l}40^{\circ} 83^{\prime} 07^{\prime \prime} \\
14^{\circ} 25^{\prime} 28^{\prime \prime}\end{array}$ \\
\hline $\mathrm{H} 4$ & Herbs & Unburnt & $\begin{array}{l}40^{\circ} 81^{\prime} 81^{\prime \prime} \\
14^{\circ} 43^{\prime} 50^{\prime \prime}\end{array}$ \\
\hline H5 & Herbs & Unburnt & $\begin{array}{l}40^{\circ} 82^{\prime} 17^{\prime \prime} \\
14^{\circ} 43^{\prime} 57^{\prime \prime}\end{array}$ \\
\hline H6 & Herbs & Burnt & $\begin{array}{l}40^{\circ} 82^{\prime} 65^{\prime \prime} \\
14^{\circ} 43^{\prime} 41^{\prime \prime}\end{array}$ \\
\hline
\end{tabular}

\subsection{Soil Analyses}

In the laboratory, the soil samples were sieved $(<2 \mathrm{~mm})$ and analyzed for $\mathrm{pH}$, water (WC), and organic matter (OM) contents, total concentration of carbon (C) and nitrogen $(\mathrm{N})$, and available fraction of $\mathrm{Al}, \mathrm{Ca}, \mathrm{Cu}, \mathrm{Fe}, \mathrm{Mg}, \mathrm{Mn}, \mathrm{Na}$, and $\mathrm{Pb}$. Soil $\mathrm{pH}$ was measured with a $\mathrm{pH}$ meter on aqueous extract obtained by adding distilled water to soil $(2.5: 1=\mathrm{w}: \mathrm{w})$, whereas WC was determined gravimetrically by drying fresh soil at $105^{\circ} \mathrm{C}$ until reaching constant weight. Total $\mathrm{C}$ and $\mathrm{N}$ concentrations were determined by gas-chromatography (Thermo Finnigan, CNS Analyzer) on dried and pulverized (Fritsch Analysette Spartan 3 Pulverisette 0 ) samples. OM was calculated multiplying by 1.724 the $\mathrm{C}_{\text {org }}$ determined 
by gas-chromatography (Thermo Finnigan, CNS Analyzer) on samples previously treated with $\mathrm{HCl}(10 \%$, v:v).

Available fraction of $\mathrm{Al}, \mathrm{Ca}, \mathrm{Cu}, \mathrm{Fe}, \mathrm{Mg}, \mathrm{Mn}, \mathrm{Na}$, and $\mathrm{Pb}$ was assessed on air-dried soil samples with diethylenetriamine pentacetic acid, $\mathrm{CaCl}_{2}$, and triethanolamine at $\mathrm{pH} 7.3 \pm 0.05$ according to Lindsay and Norwell [33]. The element concentrations were measured by Inductively Coupled Plasma Mass Spectrometry (ICP-MS Aurora M90, Bruker). Accuracy of element measurements was checked by concurrent analysis of standard reference material (BCR CRM 142R - Commission of the European Communities, 1994). The overall element recovery ranged from $80 \%-120 \%$ for all the investigated soil samples.

Hydrolase activity (HA) was determined by adding to $1 \mathrm{~g}$ of fresh soil $7.5 \mathrm{~mL}$ of $60 \mathrm{mM}$ potassium phosphate at $\mathrm{pH} 7.6$ and $0.100 \mathrm{~mL}$ of fluorescein diacetate (FDA). The reaction mixture was incubated at $30^{\circ} \mathrm{C}$ for $20 \mathrm{~min}$, after which the fluorescein was extracted with $7.5 \mathrm{~mL}$ of acetone. The extract was centrifuged at $5000 \mathrm{rpm}$ for $5 \mathrm{~min}$ and the absorbance of the supernatant was measured at $490 \mathrm{~nm}$ and the results were expressed as mmol of FDA produced for $1 \mathrm{~g}$ of dry soil in $1 \mathrm{~h}$ [25]. Dehydrogenase activity (DHA) was determined by adding to $1 \mathrm{~g}$ of fresh soil $1 \mathrm{~mL}$ of 1.5\% 2,3,5-triphenyltetrazolium chloride (TTC) dissolved in $0.1 \mathrm{M}$ Tris- $\mathrm{HCl}$ buffer at $\mathrm{pH} 7.5$. The reaction mixture was incubated at $30^{\circ} \mathrm{C}$ for $24 \mathrm{~h}$ in the dark, after which the triphenylformazan (TFF) was extracted with $8 \mathrm{~mL}$ of acetone. The extract was centrifuged at $3500 \mathrm{rpm}$ for $15 \mathrm{~min}$ and the absorbance of the supernatant was measured at $546 \mathrm{~nm}$ and the results were expressed as mmol of TFF produced for $1 \mathrm{~g}$ of dry soil in $1 \mathrm{~h}$ [15]. $\beta$-glucosidase activity ( $\beta$-GLU) was determined by adding to $1 \mathrm{~g}$ of fresh soil $4 \mathrm{~mL}$ of modified universal buffer (MUB) $\mathrm{pH} 6$ and $1 \mathrm{~mL}$ of $0.025 \mathrm{M}$ p-nitrophenyl $\beta$-D-glucopyranoside (PNP). The reaction mixture was incubated at $37{ }^{\circ} \mathrm{C}$ for $1 \mathrm{~h}$, after which the enzymatic reaction was stopped by cooling on ice for $15 \mathrm{~min}$. Then, $1 \mathrm{~mL}$ of $0.5 \mathrm{M} \mathrm{CaCl}_{2}$ and $4 \mathrm{~mL}$ of $0.1 \mathrm{M}$ tris-hydroxymethilaminomethane-sodium hydroxide (THAM-NaOH) pH 12 was added. The extract was centrifuged at $5000 \mathrm{rpm}$ for $15 \mathrm{~min}$ and the absorbance of the supernatant was measured at $420 \mathrm{~nm}$ and the results were expressed as mmol of PNP produced for $1 \mathrm{~g}$ of dry soil in $1 \mathrm{~h}[34,35]$.

\subsection{Statistical Analyses}

The normality of the distribution of the data sets was assessed by the Shapiro-Wilk test.

According to the data distribution, in order to compare the differences among different seasons, summer (S) and fall (F) 2018, and winter (W) and spring (Sp) 2019 within each vegetation cover (P: pine; HO: holm oak; BL: black locust; $\mathrm{H}$ : herbs) for both the soil abiotic and biotic properties the ANOVA tests were performed.

In order to compare the differences between unburnt (UB) and burnt (B) soils within each vegetation cover $(\mathrm{P}, \mathrm{HO}, \mathrm{BL}$, and $\mathrm{H})$ for both the soil abiotic and biotic properties, the $t$-tests were performed.

In order to highlight the variability of the data during the investigated period and due to fire occurrence, the coefficients of variations (CVs) were calculated as the ratio between standard deviation and mean value for each enzymatic activity.

In order to assess the linear significant impacts of soil abiotic properties on soil biotic ones, multiple linear regressions were carried out with $\mathrm{lm}$ package of $\mathrm{R}$ 4.0.3 programming. The responses of soil biotic properties (HA, DHA, and $\beta-G L U)$, defined as dependent variables to soil abiotic properties $(\mathrm{pH}, \mathrm{WC}$, total $\mathrm{C}$, and $\mathrm{N}$ concentrations, $\mathrm{OM}$, and available fraction of $\mathrm{Al}, \mathrm{Ca}, \mathrm{Cu}, \mathrm{Fe}, \mathrm{Mg}, \mathrm{Mn}$, and $\mathrm{Na}$ and $\mathrm{Pb}$ ), defined as independent variables, were performed. To perform the multiple linear regressions, collinearity among independent variables was checked and the collinear variables were excluded from the model. Before performing the multiple linear regressions, the linearity of the data, the independence, the homogeneity, and the normality of residuals were tested. The models were considered significant at least for $p<0.05$.

In order to identify the relationships among soil enzymatic activities and abiotic properties within each vegetation cover, four Principal Components Analyses (PCA) were 
performed. In addition, to highlight the significant differences among different seasons (S, F, W, and Sp), and between unburnt and burnt soils within the same vegetation cover, permutational multivariate analyses (PERMANOVA) were performed.

All the statistical analyses were performed using the $\mathrm{R}$ 4.0.3 programming environment with vegan package [36]. All the statistical analyses were considered significant at least for $p<0.05$.

The graphs were created with SigmaPlot12 software (Jandel Scientific, San Rafael, CA, USA).

\section{Results}

Soil Abiotic and Biotic Properties within the Same Vegetation Cover

The values of the investigated abiotic properties of the soils collected at the various seasons under the different vegetation covers are reported in Table 1 . The soils under pine showed the higher cases of significant differences among the seasons, especially for the element availabilities (Table 2).

Table 2. Abiotic properties of soils under different vegetation covers in each season. Mean values $(n=6, \pm$ s.e.) of $\mathrm{pH}$, water content (WC, expressed as \% d.w), C and N concentrations (expressed as \% d.w.), organic matter content (OM, expressed as \% d.w.), and $\mathrm{Al}, \mathrm{Ca}, \mathrm{Cu}, \mathrm{Fe}, \mathrm{K}, \mathrm{Mg}, \mathrm{Mn}, \mathrm{Na}$, and $\mathrm{Pb}$ available fractions (expressed as $\mu \mathrm{g} \mathrm{g}^{-1} \mathrm{~d} . \mathrm{w}$.) in soils collected inside the Vesuvius National Park under different vegetation covers (P: pine; HO: holm oak; BL: black locust; H: herbs) and seasons (S: summer 2018; F: fall 2018; W: winter 2019; Sp: spring 2019). Different letters indicate statistically significant differences among seasons in each property of soils collected under the same vegetation cover (ANOVA test, at least, $p<0.05)$.

\begin{tabular}{|c|c|c|c|c|c|c|c|c|c|c|c|c|c|c|c|c|}
\hline & \multicolumn{4}{|c|}{$\mathbf{P}$} & \multicolumn{4}{|c|}{ HO } & \multicolumn{4}{|c|}{ BL } & \multicolumn{4}{|c|}{$\mathbf{H}$} \\
\hline & $S$ & $\mathbf{F}$ & W & $\mathrm{Sp}$ & S & F & W & $\mathrm{Sp}$ & $S$ & F & W & $\mathrm{Sp}$ & $\mathrm{S}$ & $F$ & W & $\mathrm{Sp}$ \\
\hline $\mathrm{pH}$ & $\begin{array}{c}7.8 a \\
\pm 0.05\end{array}$ & $\begin{array}{l}7.36 b \\
\pm 0.09\end{array}$ & $\begin{array}{l}7.06 b \\
\pm 0.30\end{array}$ & $\begin{array}{c}7.54 \\
a b \\
\pm 0.17\end{array}$ & $\begin{array}{c}7.35 \\
\pm 0.28\end{array}$ & $\begin{array}{c}7.13 \\
\pm 0.25\end{array}$ & $\begin{array}{c}7.34 \\
\pm 0.25\end{array}$ & $\begin{array}{c}7.55 \\
\pm 0.13\end{array}$ & $\begin{array}{c}7.10 \\
a b \\
\pm 0.11\end{array}$ & $\begin{array}{c}6.91 \\
a b \\
\pm 0.12\end{array}$ & $\begin{array}{l}6.91 b \\
\pm 0.05\end{array}$ & $\begin{array}{l}7.29 a \\
\pm 0.08\end{array}$ & $\begin{array}{c}7.46 \\
\pm 0.11\end{array}$ & $\begin{array}{c}7.53 \\
\pm 0.04\end{array}$ & $\begin{array}{c}7.27 \\
\pm 0.09\end{array}$ & $\begin{array}{c}7.56 \\
\pm 0.15\end{array}$ \\
\hline WC & $\begin{array}{l}6.14 b \\
\pm 1.28\end{array}$ & $\begin{array}{c}12.9 \\
a b \\
\pm 2.61\end{array}$ & $\begin{array}{l}16.9 a \\
\pm 0.35\end{array}$ & $\begin{array}{c}8.94 \\
a b \\
\pm 3.01\end{array}$ & $\begin{array}{c}27.7 \\
a b \\
\pm 8.27\end{array}$ & $\begin{array}{l}26.3 b \\
\pm 3.07\end{array}$ & $\begin{array}{l}46.5 a \\
\pm 4.34\end{array}$ & $\begin{array}{l}23.1 b \\
\pm 3.91\end{array}$ & $\begin{array}{l}3.54 b \\
\pm 0.96\end{array}$ & $\begin{array}{l}11.7 a \\
\pm 1.64\end{array}$ & $\begin{array}{l}17.0 a \\
\pm 0.85\end{array}$ & $\begin{array}{l}5.34 b \\
\pm 0.98\end{array}$ & $\begin{array}{c}5.60 \\
\pm 3.48\end{array}$ & $\begin{array}{c}13.6 \\
\pm 5.22\end{array}$ & $\begin{array}{c}13.7 \\
\pm 6.95\end{array}$ & $\begin{array}{c}4.20 \\
\pm 1.09\end{array}$ \\
\hline C & $\begin{array}{c}2.71 \\
\pm 0.43\end{array}$ & $\begin{array}{c}3.24 \\
\pm 0.25\end{array}$ & $\begin{array}{c}2.87 \\
\pm 0.11 \\
\end{array}$ & $\begin{array}{c}3.15 \\
\pm 0.75\end{array}$ & $\begin{array}{c}7.68 \\
\pm 3.23\end{array}$ & $\begin{array}{c}6.94 \\
\pm 1.94\end{array}$ & $\begin{array}{c}8.96 \\
\pm 1.39\end{array}$ & $\begin{array}{c}8.27 \\
\pm 1.22\end{array}$ & $\begin{array}{c}7.35 \\
\pm 1.88\end{array}$ & $\begin{array}{c}5.69 \\
\pm 2.33\end{array}$ & $\begin{array}{c}3.54 \\
\pm 0.41\end{array}$ & $\begin{array}{c}1.82 \\
\pm 0.74\end{array}$ & $\begin{array}{c}2.32 \\
\pm 0.95\end{array}$ & $\begin{array}{c}1.70 \\
\pm 1.16\end{array}$ & $\begin{array}{c}2.18 \\
\pm 1.92 \\
\end{array}$ & $\begin{array}{c}2.48 \\
\pm 1.23\end{array}$ \\
\hline $\mathbf{N}$ & $\begin{array}{c}0.23 \\
\pm 0.02\end{array}$ & $\begin{array}{c}0.20 \\
\pm 0.05\end{array}$ & $\begin{array}{c}0.18 \\
\pm 0.03\end{array}$ & $\begin{array}{c}0.18 \\
\pm 0.05\end{array}$ & $\begin{array}{c}0.51 \\
\pm 0.16\end{array}$ & $\begin{array}{c}0.54 \\
\pm 0.19\end{array}$ & $\begin{array}{c}0.57 \\
\pm 0.10\end{array}$ & $\begin{array}{c}0.49 \\
\pm 0.07\end{array}$ & $\begin{array}{c}1.09 \\
\pm 0.53\end{array}$ & $\begin{array}{c}0.62 \\
\pm 0.25\end{array}$ & $\begin{array}{c}0.36 \\
\pm 0.03\end{array}$ & $\begin{array}{c}0.76 \\
\pm 0.57\end{array}$ & $\begin{array}{c}0.29 \\
\pm 0.13\end{array}$ & $\begin{array}{c}0.27 \\
\pm 0.09\end{array}$ & $\begin{array}{c}0.10 \\
\pm 0.05\end{array}$ & $\begin{array}{c}0.17 \\
\pm 0.08\end{array}$ \\
\hline OM & $\begin{array}{c}4.82 \\
\pm 2.26\end{array}$ & $\begin{array}{c}4.27 \\
\pm 1.75\end{array}$ & $\begin{array}{c}4.46 \\
\pm 2.10\end{array}$ & $\begin{array}{c}4.46 \\
\pm 2.73\end{array}$ & $\begin{array}{c}5.37 \\
\pm 0.78\end{array}$ & $\begin{array}{c}5.79 \\
\pm 1.37\end{array}$ & $\begin{array}{c}6.40 \\
\pm 1.45\end{array}$ & $\begin{array}{c}5.50 \\
\pm 0.47\end{array}$ & $\begin{array}{c}10.9 \\
\pm 3.54\end{array}$ & $\begin{array}{c}12.6 \\
\pm 3.63\end{array}$ & $\begin{array}{c}11.3 \\
\pm 3.01\end{array}$ & $\begin{array}{c}8.68 \\
\pm 1.99\end{array}$ & $\begin{array}{c}8.32 \\
\pm 2.52\end{array}$ & $\begin{array}{c}8.07 \\
\pm 2.26\end{array}$ & $\begin{array}{c}7.98 \\
\pm 1.50\end{array}$ & $\begin{array}{c}6.29 \\
\pm 1.89\end{array}$ \\
\hline Al & $\begin{array}{l}5.15 c \\
\pm 1.08\end{array}$ & $\begin{array}{l}17.2 b \\
\pm 1.41 \\
\end{array}$ & $\begin{array}{r}25.6 a \\
\pm 2.22 \\
\end{array}$ & $\begin{array}{l}10.1 c \\
\pm 2.20\end{array}$ & $\begin{array}{l}7.09 b \\
\pm 1.10\end{array}$ & $\begin{array}{c}25.2 \\
a b \\
\pm 7.77\end{array}$ & $\begin{array}{l}29.9 a \\
\pm 15.5\end{array}$ & $\begin{array}{c}11.3 \\
a b \\
\pm 2.98\end{array}$ & $\begin{array}{l}6.76 c \\
\pm 2.19\end{array}$ & $\begin{array}{c}14.4 \\
a c \\
\pm 1.74\end{array}$ & $\begin{array}{l}19.9 a \\
\pm 1.58\end{array}$ & $\begin{array}{l}8.41 b \\
\pm 1.28\end{array}$ & $\begin{array}{l}2.67 b \\
\pm 0.59\end{array}$ & $\begin{array}{l}7.53 a \\
\pm 1.19\end{array}$ & $\begin{array}{l}9.89 a \\
\pm 1.75\end{array}$ & $\begin{array}{c}4.33 \\
a b \\
\pm 1.23\end{array}$ \\
\hline $\mathrm{Ca}$ & $\begin{array}{c}3730 b \\
\pm 650\end{array}$ & $\begin{array}{r}5983 a \\
\pm 705\end{array}$ & $\begin{array}{c}2078 b \\
\pm 201\end{array}$ & $\begin{array}{c}4541 \\
a b \\
\pm 971\end{array}$ & $\begin{array}{c}8353 \\
\pm 1844\end{array}$ & $\begin{array}{l}13,550 \\
\pm 3428\end{array}$ & $\begin{array}{c}8275 \\
\pm 1749\end{array}$ & $\begin{array}{l}12,413 \\
\pm 2165\end{array}$ & $\begin{array}{c}5013 \\
\pm 1501\end{array}$ & $\begin{array}{c}8083 \\
\pm 2123\end{array}$ & $\begin{array}{c}3581 \\
\pm 765\end{array}$ & $\begin{array}{c}4748 \\
\pm 882\end{array}$ & $\begin{array}{c}3021 \\
\pm 1218\end{array}$ & $\begin{array}{c}5432 \\
\pm 3555\end{array}$ & $\begin{array}{c}6551 \\
\pm 3598\end{array}$ & $\begin{array}{c}6864 \\
\pm 4558\end{array}$ \\
\hline $\mathrm{Cu}$ & $\begin{array}{l}0.72 b \\
\pm 0.20\end{array}$ & $\begin{array}{c}2.02 \\
a b \\
\pm 0.69\end{array}$ & $\begin{array}{l}2.18 a \\
\pm 0.09 \\
\end{array}$ & $\begin{array}{l}1.49 b \\
\pm 0.25\end{array}$ & $\begin{array}{l}0.64 b \\
\pm 0.10\end{array}$ & $\begin{array}{l}1.50 a \\
\pm 0.18 \\
\end{array}$ & $\begin{array}{l}1.99 a \\
\pm 0.46\end{array}$ & $\begin{array}{l}1.85 a \\
\pm 0.30\end{array}$ & $\begin{array}{l}0.72 b \\
\pm 0.14 \\
\end{array}$ & $\begin{array}{l}2.44 a \\
\pm 0.45\end{array}$ & $\begin{array}{l}3.32 a \\
\pm 1.15\end{array}$ & $\begin{array}{c}1.23 \\
a b \\
\pm 0.19\end{array}$ & $\begin{array}{l}0.57 b \\
\pm 0.14\end{array}$ & $\begin{array}{l}2.58 a \\
\pm 0.44 \\
\end{array}$ & $\begin{array}{l}1.97 a \\
\pm 0.50\end{array}$ & $\begin{array}{c}1.60 \\
a b \\
\pm 0.50\end{array}$ \\
\hline $\mathrm{Fe}$ & $\begin{array}{l}8.75 c \\
\pm 0.85\end{array}$ & $\begin{array}{c}35.6 \\
a b \\
\pm 3.88\end{array}$ & $\begin{array}{l}38.8 a \\
\pm 3.48\end{array}$ & $\begin{array}{c}18.8 \\
b c \\
\pm 3.93\end{array}$ & $\begin{array}{l}25.6 c \\
\pm 5.88\end{array}$ & $\begin{array}{c}68.5 \\
a b \\
\pm 7.71\end{array}$ & $\begin{array}{l}95.1 a \\
\pm 12.4\end{array}$ & $\begin{array}{c}42.9 \\
b c \\
\pm 5.97\end{array}$ & $\begin{array}{l}12.4 b \\
\pm 2.59\end{array}$ & $\begin{array}{c}24.1 \\
a b \\
\pm 5.06\end{array}$ & $\begin{array}{l}38.3 a \\
\pm 9.57\end{array}$ & $\begin{array}{c}13.2 \\
a b \\
\pm 2.79\end{array}$ & $\begin{array}{c}5.98 \\
\pm 1.57\end{array}$ & $\begin{array}{c}22.6 \\
\pm 8.75\end{array}$ & $\begin{array}{c}20.1 \\
\pm 12.6\end{array}$ & $\begin{array}{c}7.29 \\
\pm 2.75\end{array}$ \\
\hline $\mathbf{K}$ & $\begin{array}{l}410 a b \\
\pm 72.7\end{array}$ & $\begin{array}{l}414 a \\
\pm 44.2\end{array}$ & $\begin{array}{l}225 b \\
\pm 14.7\end{array}$ & $\begin{array}{l}376 a b \\
\pm 69.7\end{array}$ & $\begin{array}{c}718 \\
\pm 98.4\end{array}$ & $\begin{array}{c}688 \\
\pm 102\end{array}$ & $\begin{array}{c}526 \\
\pm 45.4\end{array}$ & $\begin{array}{c}740 \\
\pm 54.2\end{array}$ & $\begin{array}{c}394 \\
\pm 72.1\end{array}$ & $\begin{array}{c}370 \\
\pm 42.3\end{array}$ & $\begin{array}{c}256 \\
\pm 20.4\end{array}$ & $\begin{array}{c}350 \\
\pm 39.9\end{array}$ & $\begin{array}{c}545 \\
\pm 206\end{array}$ & $\begin{array}{c}438 \\
\pm 126\end{array}$ & $\begin{array}{c}394 \\
\pm 131\end{array}$ & $\begin{array}{c}668 \\
\pm 327\end{array}$ \\
\hline $\mathrm{Mg}$ & $\begin{array}{c}293 b \\
\pm 89.4\end{array}$ & $\begin{array}{r}449 a \\
\pm 32.5\end{array}$ & $\begin{array}{c}195 c \\
\pm 13.2\end{array}$ & $\begin{array}{l}40.5 d \\
\pm 2.11\end{array}$ & $\begin{array}{c}608 a \\
\pm 92.1\end{array}$ & $\begin{array}{l}836 \text { a } \\
\pm 173\end{array}$ & $\begin{array}{c}447 a \\
\pm 57.8\end{array}$ & $\begin{array}{l}48.4 b \\
\pm 2.74\end{array}$ & $\begin{array}{c}360 a \\
\pm 78.8\end{array}$ & $\begin{array}{l}517 a \\
\pm 99.4\end{array}$ & $\begin{array}{l}273 a \\
\pm 41.0\end{array}$ & $\begin{array}{l}38.9 b \\
\pm 2.40\end{array}$ & $\begin{array}{c}188 a \\
\pm 44.2\end{array}$ & $\begin{array}{l}290 a \\
\pm 122\end{array}$ & $\begin{array}{l}299 a \\
\pm 123\end{array}$ & $\begin{array}{c}33.8 \\
a b \\
\pm 33.5\end{array}$ \\
\hline Mn & $\begin{array}{l}1.03 b \\
\pm 0.11\end{array}$ & $\begin{array}{l}3.77 a \\
\pm 0.61\end{array}$ & $\begin{array}{l}3.58 a \\
\pm 0.38\end{array}$ & $\begin{array}{l}3.98 a \\
\pm 0.66\end{array}$ & $\begin{array}{c}4.98 \\
\pm 2.26\end{array}$ & $\begin{array}{c}14.3 \\
\pm 3.19\end{array}$ & $\begin{array}{c}17.6 \\
\pm 2.86\end{array}$ & $\begin{array}{c}24.3 \\
\pm 7.09\end{array}$ & $\begin{array}{c}5.66 \\
\pm 2.44\end{array}$ & $\begin{array}{c}7.09 \\
\pm 2.64\end{array}$ & $\begin{array}{c}6.49 \\
\pm 0.96\end{array}$ & $\begin{array}{c}3.37 \\
\pm 0.99\end{array}$ & $\begin{array}{c}0.44 \\
\pm 0.09\end{array}$ & $\begin{array}{c}1.42 \\
\pm 1.05\end{array}$ & $\begin{array}{c}3.16 \\
\pm 2.39\end{array}$ & $\begin{array}{c}1.31 \\
\pm 0.92\end{array}$ \\
\hline $\mathrm{Na}$ & $\begin{array}{l}514 b \\
\pm 49.1\end{array}$ & $\begin{array}{l}874 a \\
\pm 46.1\end{array}$ & $\begin{array}{l}848 a \\
\pm 23.5\end{array}$ & $\begin{array}{c}833 a b \\
\pm 155\end{array}$ & $\begin{array}{l}585 c \\
\pm 75.4\end{array}$ & $\begin{array}{l}922 a b \\
\pm 48.0\end{array}$ & $\begin{array}{l}946 b \\
\pm 23.2\end{array}$ & $\begin{array}{l}1098 a \\
\pm 50.4\end{array}$ & $\begin{array}{l}482 c \\
\pm 40.4\end{array}$ & $\begin{array}{l}917 b \\
\pm 44.8\end{array}$ & $\begin{array}{l}958 a b \\
\pm 28.0\end{array}$ & $\begin{array}{l}1078 a \\
\pm 26.2\end{array}$ & $\begin{array}{l}514 b \\
\pm 58.9\end{array}$ & $\begin{array}{l}908 a \\
\pm 47.7\end{array}$ & $\begin{array}{l}937 a \\
\pm 44.9\end{array}$ & $\begin{array}{c}1284 a \\
\pm 198\end{array}$ \\
\hline $\mathrm{Pb}$ & $\begin{array}{l}0.81 b \\
\pm 0.12\end{array}$ & $\begin{array}{l}2.41 a \\
\pm 0.65\end{array}$ & $\begin{array}{l}2.26 a \\
\pm 0.74\end{array}$ & $\begin{array}{l}2.43 a \\
\pm 0.58\end{array}$ & $\begin{array}{l}2.00 \mathrm{~b} \\
\pm 0.54\end{array}$ & $\begin{array}{l}2.50 \mathrm{~b} \\
\pm 1.04\end{array}$ & $\begin{array}{l}2.75 b \\
\pm 1.26\end{array}$ & $\begin{array}{l}4.61 a \\
\pm 0.55\end{array}$ & $\begin{array}{l}0.53 b \\
\pm 0.17\end{array}$ & $\begin{array}{l}3.80 a \\
\pm 1.46\end{array}$ & $\begin{array}{l}6.65 a \\
\pm 3.21\end{array}$ & $\begin{array}{l}0.53 b \\
\pm 0.15\end{array}$ & $\begin{array}{c}0.48 \\
\pm 0.28\end{array}$ & $\begin{array}{c}2.69 \\
\pm 1.13\end{array}$ & $\begin{array}{c}2.31 \\
\pm 0.80\end{array}$ & $\begin{array}{c}2.15 \\
\pm 1.33\end{array}$ \\
\hline
\end{tabular}


The values of the investigated abiotic properties in unburnt and burnt soils under the different vegetation covers are reported in Table 3. The significant differences between unburnt and burnt soils under the same vegetation cover were few (Table 3).

Table 3. Abiotic properties of unburnt and burnt soils under different vegetation covers. Mean values $(n=12, \pm$ s.e.) of $\mathrm{pH}$, water content (WC, expressed as $\% \mathrm{~d} . \mathrm{w}), \mathrm{C}$ and $\mathrm{N}$ concentrations (expressed as \% d.w.), organic matter content (OM, expressed as \% d.w.), and $\mathrm{Al}, \mathrm{Ca}, \mathrm{Cu}, \mathrm{Fe}, \mathrm{K}, \mathrm{Mg}$, $\mathrm{Mn}, \mathrm{Na}$, and $\mathrm{Pb}$ available fractions (expressed as $\mu \mathrm{g} \mathrm{g}^{-1} \mathrm{~d} . \mathrm{w}$.) in unburnt (UB) and burnt (B) soils collected inside the Vesuvius National Park under different vegetation covers (P: pine; HO: holm oak; BL: black locust; H: herbs). Different letters indicate statistically significant differences in each property between unburnt and burnt soils under the same vegetation cover $(t$-test, at least, $p<0.05)$.

\begin{tabular}{|c|c|c|c|c|c|c|c|c|}
\hline & \multicolumn{2}{|c|}{$\mathbf{P}$} & \multicolumn{2}{|c|}{ HO } & \multicolumn{2}{|c|}{ BL } & \multicolumn{2}{|c|}{$\mathbf{H}$} \\
\hline & UB & B & UB & B & UB & B & UB & B \\
\hline $\mathrm{pH}$ & $\begin{array}{c}7.48 \\
\pm 0.17\end{array}$ & $\begin{array}{c}7.43 \\
\pm 0.12\end{array}$ & $\begin{array}{c}7.43 \\
\pm 0.15\end{array}$ & $\begin{array}{c}7.21 \\
\pm 0.14\end{array}$ & $\begin{array}{c}7.08 \\
\pm 0.05\end{array}$ & $\begin{array}{c}7.03 \\
\pm 0.08\end{array}$ & $\begin{array}{l}7.58 a \\
\pm 0.06\end{array}$ & $\begin{array}{l}7.27 b \\
\pm 0.05\end{array}$ \\
\hline WC & $\begin{array}{c}11.5 \\
\pm 2.19\end{array}$ & $\begin{array}{c}11.2 \\
\pm 1.61\end{array}$ & $\begin{array}{c}34.7 \\
\pm 4.36\end{array}$ & $\begin{array}{c}25.1 \\
\pm 4.12\end{array}$ & $\begin{array}{c}10 \\
\pm 2.23\end{array}$ & $\begin{array}{c}8.96 \\
\pm 1.73\end{array}$ & $\begin{array}{c}11.9 \\
\pm 3.75\end{array}$ & $\begin{array}{l}5.34 \\
\pm 1.36\end{array}$ \\
\hline C & $\begin{array}{c}3.03 \\
\pm 0.32\end{array}$ & $\begin{array}{c}2.98 \\
\pm 0.26\end{array}$ & $\begin{array}{c}9.47 \\
\pm 1.32\end{array}$ & $\begin{array}{c}5.69 \\
\pm 1.13\end{array}$ & $\begin{array}{c}3.35 \\
\pm 0.65\end{array}$ & $\begin{array}{c}5.43 \\
\pm 1.34\end{array}$ & $\begin{array}{c}2.79 \\
\pm 0.98\end{array}$ & $\begin{array}{c}1.25 \\
\pm 0.49\end{array}$ \\
\hline $\mathbf{N}$ & $\begin{array}{c}0.15 \\
\pm 0.03\end{array}$ & $\begin{array}{c}0.20 \\
\pm 0.02\end{array}$ & $\begin{array}{c}0.61 \\
\pm 0.09\end{array}$ & $\begin{array}{c}0.39 \\
\pm 0.04\end{array}$ & $\begin{array}{c}0.66 \\
\pm 0.34\end{array}$ & $\begin{array}{c}0.74 \\
\pm 0.24\end{array}$ & $\begin{array}{c}0.25 \\
\pm 0.07\end{array}$ & $\begin{array}{c}0.14 \\
\pm 0.02\end{array}$ \\
\hline OM & $\begin{array}{c}3.49 \\
\pm 0.58\end{array}$ & $\begin{array}{c}4.75 \\
\pm 1.28\end{array}$ & $\begin{array}{c}5.04 \\
\pm 0.74\end{array}$ & $\begin{array}{c}6.86 \\
\pm 0.45\end{array}$ & $\begin{array}{c}10.4 \\
\pm 2.11\end{array}$ & $\begin{array}{c}11.2 \\
\pm 2.06\end{array}$ & $\begin{array}{c}8.18 \\
\pm 1.52\end{array}$ & $\begin{array}{c}6.88 \\
\pm 0.93\end{array}$ \\
\hline Al & $\begin{array}{c}14.6 \\
\pm 3.81\end{array}$ & $\begin{array}{c}14.5 \\
\pm 2.30\end{array}$ & $\begin{array}{l}11.8 b \\
\pm 3.29\end{array}$ & $\begin{array}{l}28.2 a \\
\pm 9.69\end{array}$ & $\begin{array}{c}11.2 \\
\pm 2.19\end{array}$ & $\begin{array}{c}13.2 \\
\pm 1.95\end{array}$ & $\begin{array}{c}4.75 \\
\pm 0.76\end{array}$ & $\begin{array}{c}8.13 \\
\pm 1.65\end{array}$ \\
\hline $\mathrm{Ca}$ & $\begin{array}{l}3832 \\
\pm 772\end{array}$ & $\begin{array}{l}4146 \\
\pm 542\end{array}$ & $\begin{array}{c}13,090 a \\
\pm 1553\end{array}$ & $\begin{array}{l}6985 b \\
\pm 1139\end{array}$ & $\begin{array}{c}5937 \\
\pm 1095\end{array}$ & $\begin{array}{c}4969 \\
\pm 1052\end{array}$ & $\begin{array}{c}6831 \\
\pm 2485\end{array}$ & $\begin{array}{c}3420 \\
\pm 1533\end{array}$ \\
\hline $\mathrm{Cu}$ & $\begin{array}{c}1.49 \\
\pm 0.33\end{array}$ & $\begin{array}{c}1.63 \\
\pm 0.26\end{array}$ & $\begin{array}{c}1.41 \\
\pm 0.20\end{array}$ & $\begin{array}{c}1.63 \\
\pm 0.35\end{array}$ & $\begin{array}{c}1.98 \\
\pm 0.44\end{array}$ & $\begin{array}{c}1.89 \\
\pm 0.56\end{array}$ & $\begin{array}{c}1.73 \\
\pm 0.34\end{array}$ & $\begin{array}{c}1.62 \\
\pm 0.42\end{array}$ \\
\hline $\mathrm{Fe}$ & $\begin{array}{c}18.4 \\
\pm 4.76\end{array}$ & $\begin{array}{c}27.3 \\
\pm 3.75\end{array}$ & $\begin{array}{c}59.9 \\
\pm 9.05\end{array}$ & $\begin{array}{c}55.2 \\
\pm 12.4\end{array}$ & $\begin{array}{c}25.5 \\
\pm 7.70\end{array}$ & $\begin{array}{c}19.7 \\
\pm 3.19\end{array}$ & $\begin{array}{c}18.3 \\
\pm 6.37\end{array}$ & $\begin{array}{l}7.52 \\
\pm 1.38\end{array}$ \\
\hline $\mathbf{K}$ & $\begin{array}{c}248 \\
\pm 23.6\end{array}$ & $\begin{array}{c}383 \\
\pm 35.4\end{array}$ & $\begin{array}{c}726 \\
\pm 59.5\end{array}$ & $\begin{array}{c}582 \\
\pm 37.4\end{array}$ & $\begin{array}{c}361 \\
\pm 23.9\end{array}$ & $\begin{array}{c}331 \\
\pm 38.7\end{array}$ & $\begin{array}{l}666 a \\
\pm 150\end{array}$ & $\begin{array}{l}278 b \\
\pm 56.0\end{array}$ \\
\hline $\mathrm{Mg}$ & $\begin{array}{c}206 \\
\pm 80.3\end{array}$ & $\begin{array}{c}254 \\
\pm 40.9\end{array}$ & $\begin{array}{l}546 \\
\pm 120\end{array}$ & $\begin{array}{c}393 \\
\pm 93.5\end{array}$ & $\begin{array}{c}297 \\
\pm 81.4\end{array}$ & $\begin{array}{c}298 \\
\pm 66.5\end{array}$ & $\begin{array}{c}221 \\
\pm 65.1\end{array}$ & $\begin{array}{c}175 \\
\pm 72.8\end{array}$ \\
\hline Mn & $\begin{array}{c}2.29 \\
\pm 0.56\end{array}$ & $\begin{array}{l}3.29 \\
\pm 0.41\end{array}$ & $\begin{array}{c}16.8 \\
\pm 3.64\end{array}$ & $\begin{array}{c}13.1 \\
\pm 3.36\end{array}$ & $\begin{array}{l}5.31 \\
\pm 1.4\end{array}$ & $\begin{array}{c}5.89 \\
\pm 1.31\end{array}$ & $\begin{array}{c}2.31 \\
\pm 1.14\end{array}$ & $\begin{array}{c}0.49 \\
\pm 0.09\end{array}$ \\
\hline $\mathrm{Na}$ & $\begin{array}{c}789 \\
\pm 119\end{array}$ & $\begin{array}{c}762 \\
\pm 59.3\end{array}$ & $\begin{array}{c}939 \\
\pm 55.9\end{array}$ & $\begin{array}{c}880 \\
\pm 90.7\end{array}$ & $\begin{array}{c}874 \\
\pm 97.1\end{array}$ & $\begin{array}{c}849 \\
\pm 66.6\end{array}$ & $\begin{array}{c}977 \\
\pm 119\end{array}$ & $\begin{array}{c}811 \\
\pm 87.7\end{array}$ \\
\hline $\mathbf{P b}$ & $\begin{array}{c}1.69 \\
\pm 0.37\end{array}$ & $\begin{array}{c}2.05 \\
\pm 0.37\end{array}$ & $\begin{array}{c}3.35 \\
\pm 0.61\end{array}$ & $\begin{array}{c}2.39 \\
\pm 0.74\end{array}$ & $\begin{array}{c}2.91 \\
\pm 1.31\end{array}$ & $\begin{array}{c}2.86 \\
\pm 1.46\end{array}$ & $\begin{array}{c}1.37 \\
\pm 0.59\end{array}$ & $\begin{array}{c}2.72 \\
\pm 0.79\end{array}$ \\
\hline
\end{tabular}

The enzymatic activities measured in soils collected in different seasons under the same vegetation cover are reported in Figure 1.

The hydrolase (HA) activity ranged from 187 to $2544 \mathrm{mmol} \mathrm{FDA} \mathrm{g}^{-1} \mathrm{~d} . \mathrm{w} . \mathrm{h}^{-1}$ in soils covered by pine (P), from 285 to 1842 mmol FDA g $^{-1}$ d.w. $\mathrm{h}^{-1}$ in soils covered by holm oak (HO), from 109 to 1275 mmol FDA g ${ }^{-1} \mathrm{~d} . \mathrm{w} . \mathrm{h}^{-1}$ in soils covered by black locust (BL), and from 36.2 to 1377 mmol FDA g ${ }^{-1}$ d.w. $\mathrm{h}^{-1}$ in soils covered by herbs (H). HA did not significantly vary among seasons in soils under $\mathrm{P}$ and $\mathrm{H}$ (Figure 1); whereas, it was statistically higher in summer and winter in soils covered by $\mathrm{HO}$ and statistically lower in spring in soils covered by BL (Figure 1). 

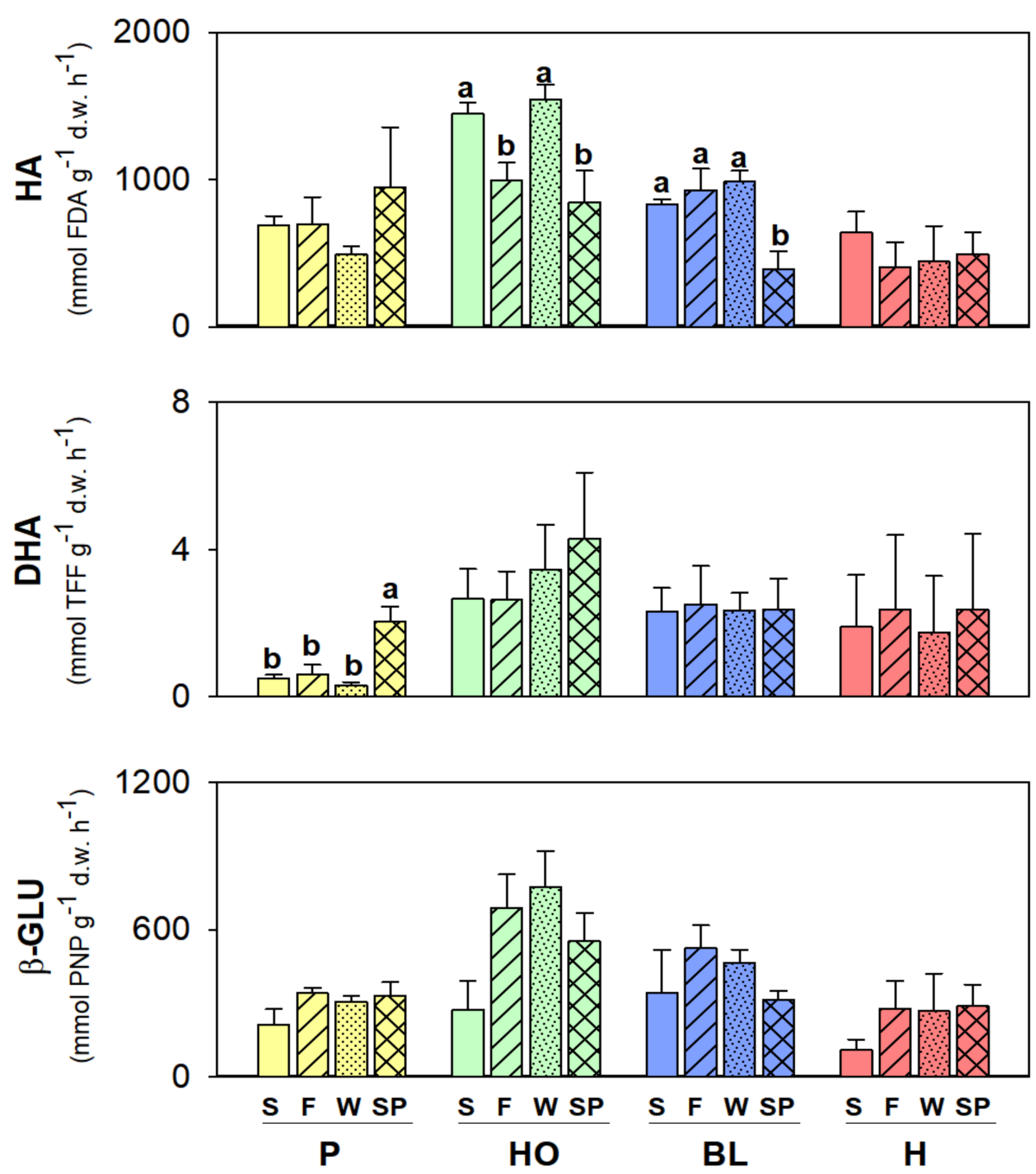

Figure 1. Mean values ( $n=6, \pm$ s.e.) of the enzymatic activities (hydrolase: HA; dehydrogenase: DHA; $\beta$-glucosidase: $\beta$-GLU) measured in surface $(0-10 \mathrm{~cm})$ soils collected inside the Vesuvius National Park (Southern Italy) under different vegetation covers (P: pine; HO: holm oak; BL: black locust; H: herbs) in summer (S) and fall (F) 2018, and winter (W) and spring (Sp) 2019. Different letters indicate statistically significant differences in enzymatic activities among different seasons under the same vegetation cover $(t$-test, at least, $p<0.05)$.

The dehydrogenase (DHA) activity ranged from 0.01 to $3.65 \mathrm{mmol} \mathrm{TFF} \mathrm{g}^{-1} \mathrm{~d} . \mathrm{w} . \mathrm{h}^{-1}$ in soils covered by pine (P), from 0.46 to $9.06 \mathrm{mmol} \mathrm{TFF}^{-1} \mathrm{~d} . \mathrm{w} . \mathrm{h}^{-1}$ in soils covered by holm oak (HO), from 0.69 to 6.47 mmol TFF $\mathrm{g}^{-1} \mathrm{~d} . \mathrm{w} . \mathrm{h}^{-1}$ in soils covered by black locust (BL), and from 0.01 to $10.6 \mathrm{mmol} \mathrm{TFF}^{-1} \mathrm{~d} . \mathrm{w} . \mathrm{h}^{-1}$ in soils covered by herbs (H). DHA statistically varied among the seasons only in soils covered by $\mathrm{P}$ with values higher in spring and lower in winter (Figure 1).

The $\beta$-glucosidase ( $\beta$-GLU) activity ranged from 63.8 to $506 \mathrm{mmol} P N P \mathrm{~g}^{-1} \mathrm{~d} . \mathrm{w} . \mathrm{h}^{-1}$ in soils covered by pine (P), from 74.6 to $1200 \mathrm{mmol} \mathrm{PNP} \mathrm{g}{ }^{-1}$ d.w. $\mathrm{h}^{-1}$ in soils covered by holm oak (HO), from 62.1 to $901 \mathrm{mmol} \mathrm{PNP} \mathrm{g}^{-1}$ d.w. $\mathrm{h}^{-1}$ in soils covered by black locust (BL), and from 61.4 to $871 \mathrm{mmol} \mathrm{PNP} \mathrm{g}^{-1}$ d.w. $\mathrm{h}^{-1}$ in soils covered by herbs (H). $\beta$-GLU did not statistically vary among the seasons in soils under the same vegetation cover (Figure 1).

The enzymatic activities (HA, DHA, and $\beta$-GLU) measured in unburnt (UB) and burnt (B) soils under the same vegetation cover are reported in Figure 2 and they did not statistically vary between UB and B soils (Figure 2). 

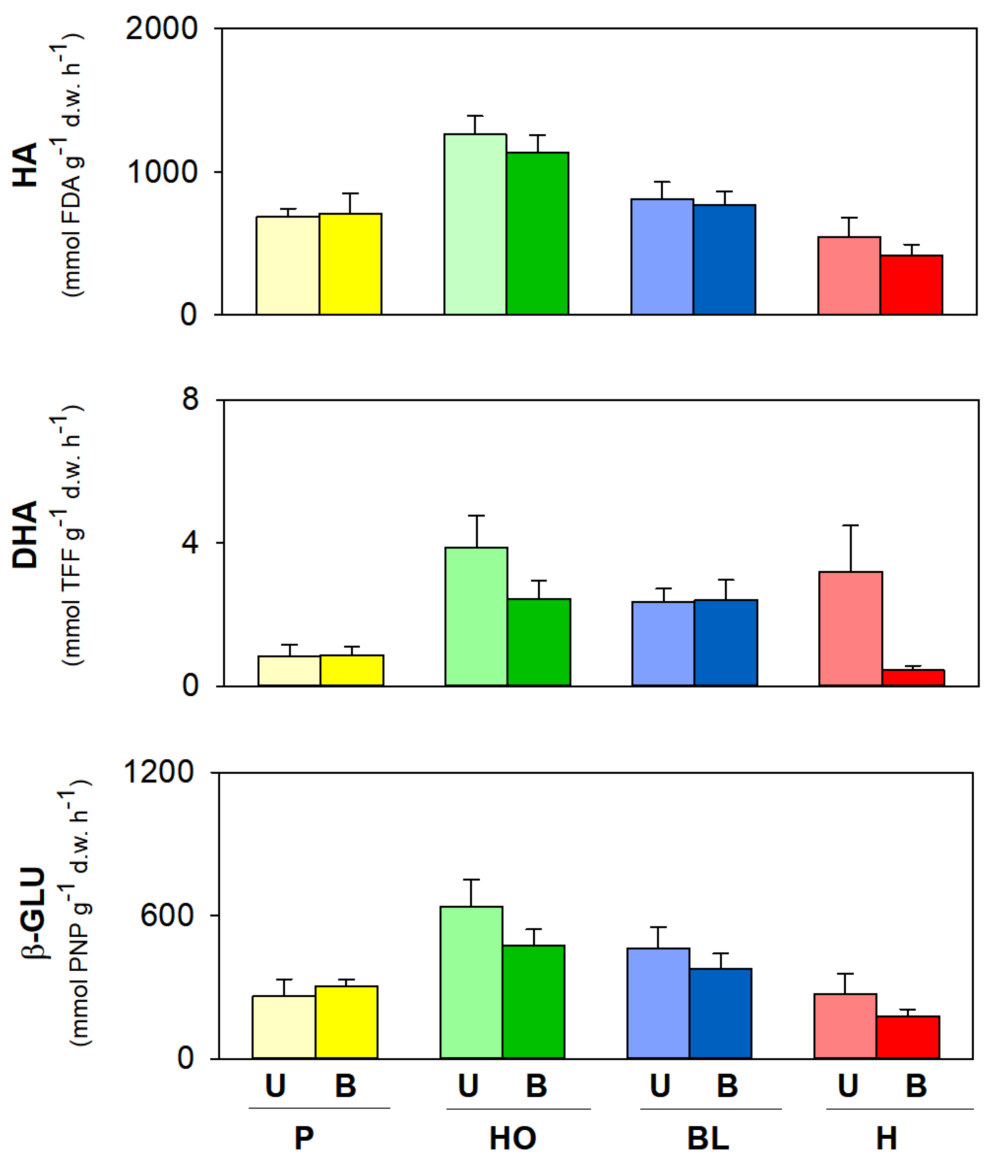

Figure 2. Mean values ( $n=12, \pm$ s.e.) of the enzymatic activities (hydrolase: HA; dehydrogenase: DHA; $\beta$-glucosidase: $\beta$-GLU) measured in surface $(0-10 \mathrm{~cm})$ soils collected inside the Vesuvius National Park (Southern Italy) in unburnt (UB) and burnt (B) soils under different vegetation covers (P: pine; HO: holm oak; BL: black locust; H: herbs).

The results of the PCA performed on soils collected under pine highlighted that the first two axes accounted, respectively, for $26 \%$ and $18 \%$ of the total variance (Figure $3 \mathrm{~A}$ ). The first axis clearly separated the soils according to the season (Figure 3A). Soil water content and available fractions of $\mathrm{Al}, \mathrm{Fe}, \mathrm{Mn}$, and $\mathrm{Na}$ were positively correlated to the first axis; whereas, HA, DHA and available fractions of $\mathrm{Ca}$ and $\mathrm{K}$ were positively correlated to the second axis (Figure 3B). The results of the PCA performed on soils collected under holm oak highlighted that the first two axes accounted, respectively, for $29 \%$ and $19 \%$ of the total variance (Figure 3B). Soil C and N, available fraction of Mn, $\beta-G L U$, and DHA were positively correlated to the first axis (Figure 3B). Available fractions of $\mathrm{Al}$, $\mathrm{Cu}$, and $\mathrm{Fe}$ were positively correlated to the second axis; whereas, available fractions of $\mathrm{K}$ and Mn concentrations were negatively correlated to it (Figure 3B). The results of the PCA performed on soils collected under black locust highlighted that the first two axes accounted, respectively, for $34 \%$ and $22 \%$ of the total variance (Figure 3C). Available fractions of $\mathrm{Ca}, \mathrm{Mg}$, and $\mathrm{Mn}$ and $\mathrm{HA}$ were positively correlated to the first axis (Figure 3C). Available fractions of $\mathrm{Al}$ and $\mathrm{Cu}$ and water content were positively correlated to the second axis; whereas, $\mathrm{C}$ and available fraction of $\mathrm{K}$ were negatively correlated to it (Figure $3 \mathrm{C}$ ). The results of the PCA performed on soils collected under herbs highlighted that the first two axes accounted, respectively, for $48 \%$ and $14 \%$ of the total variance (Figure 3D). Soil C, available fraction of $\mathrm{Ca}$, and DHA were positively correlated to the first axis (Figure 3D). Available fractions of $\mathrm{Al}, \mathrm{Cu}, \mathrm{Mg}$, and $\mathrm{Pb}$ were positively correlated to the second axis; whereas, $\mathrm{pH}$ and available fraction of $\mathrm{K}$ were negatively correlated to it (Figure 3D). 

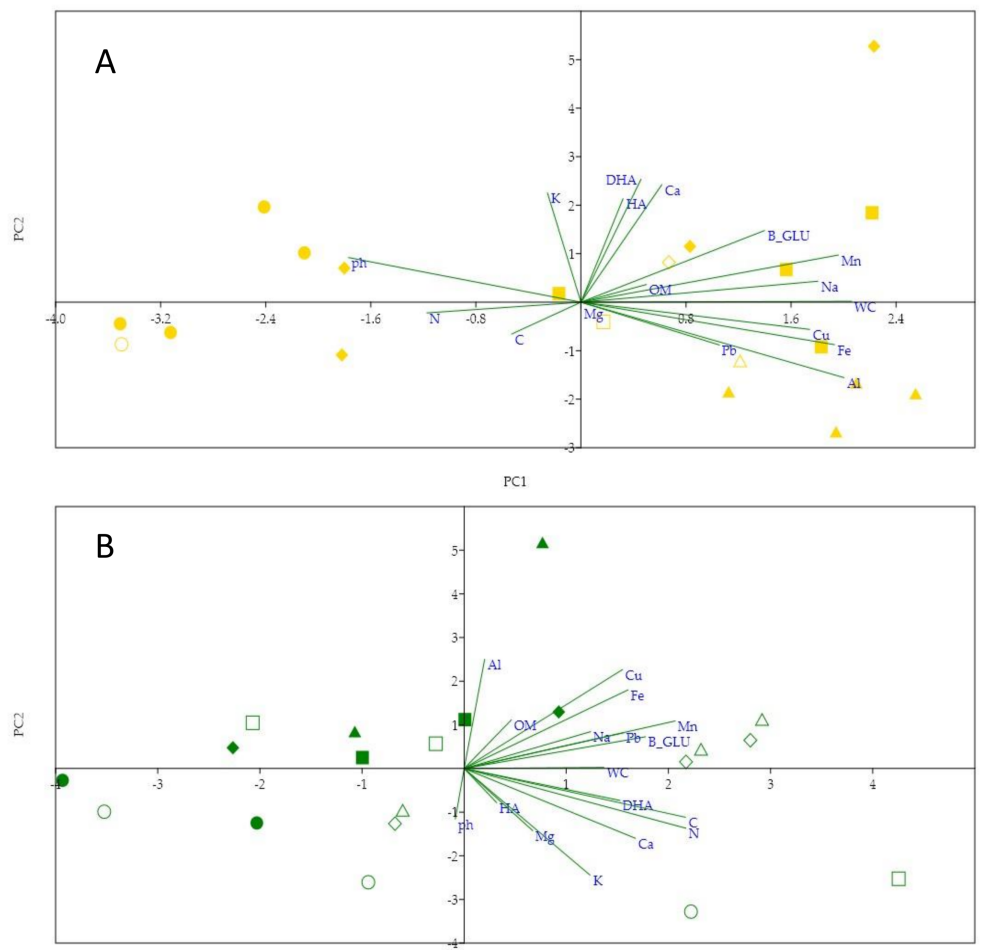

PC1

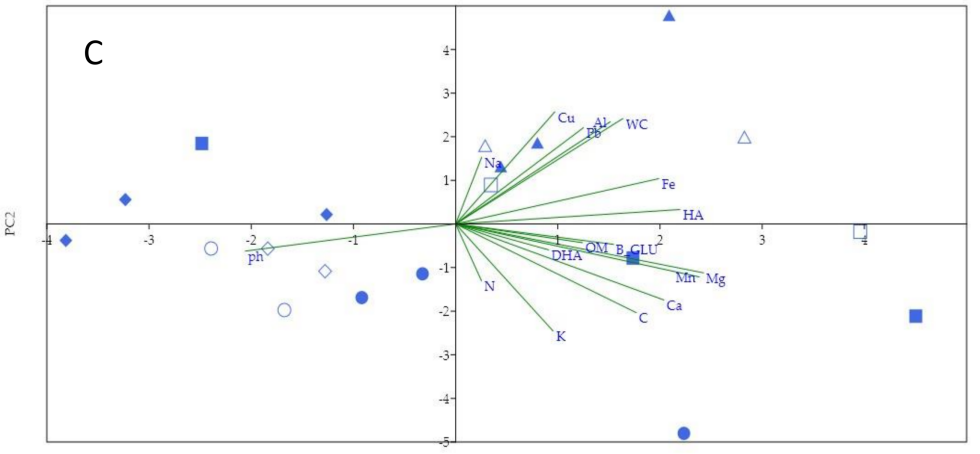

PC1

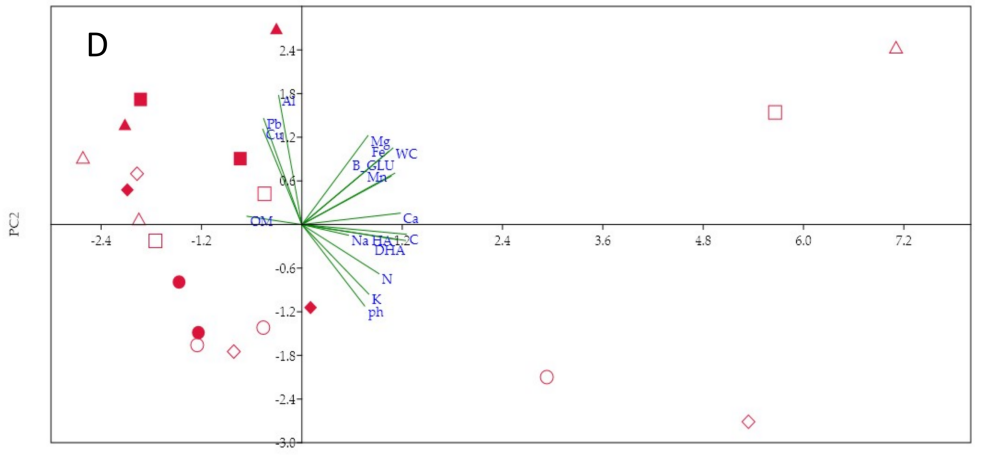

Figure 3. Graphical display of the first two axes of the Principal Component Analysis on the soil abiotic ( $\mathrm{pH}$; water content: WC; organic matter: $\mathrm{OM}$; $\mathrm{C}$ and $\mathrm{N}$, available fractions of $\mathrm{Al}, \mathrm{Ca}, \mathrm{Cu}$, $\mathrm{Fe}, \mathrm{K}, \mathrm{Mg}, \mathrm{Mn}, \mathrm{Na}$, and $\mathrm{Pb}$ ) and biotic (hydrolase: $\mathrm{HA}$; dehydrogenase: DHA and $\beta$-glucosidase: $\beta$-GLU) properties measured in unburnt (empty) and burnt (full) soils covered by pine (yellow in panel A), holm oak (green in panel B), black locust (blue in panel C), and herbs (red in panel D) in soils collected inside the Vesuvius National Park (Southern Italy) in summer (dot) and fall (square) 2018, and winter (triangle) and spring (rhombus) 2019. 
The results of PERMANOVA showed that soil abiotic and biotic properties significantly $(p=0.002)$ varied among different seasons only for soils covered by $p$ and significantly $(p=0.04)$ varied between UB and B only for soils covered by HO.

The coefficients of variation (CVs) calculated for all enzymatic activities widely varied for both seasonality and fire in soils under the investigated vegetation covers, although with different extents (Table 4). In more detail, the CVs calculated for HA showed the widest variability both for seasonality and for fire in soils covered by P (Table 4); they were wider for seasonality than for fire in soils under $\mathrm{HO}$ and BL (Table 4); conversely, they were wider for fire under $\mathrm{H}$ (Table 3).

Table 4. Coefficient of variation of the enzymatic activities. Coefficient of variation $(\mathrm{CV})$ of hydrolase (HA), dehydrogenase (DHA), and $\beta$-glucosidase ( $\beta$-GLU) activities calculated in burned and unburned (fire) soils under different vegetation covers (P: pine; HO: holm oak; BL: black locust; $\mathrm{H}$ : herbs) at different sampling seasons (S: summer 2018; F: fall 2018; W: winter 2019; SP: spring 2019).

\begin{tabular}{cccccccccc}
\hline & \multicolumn{2}{c}{ P } & \multicolumn{2}{c}{ HO } & \multicolumn{2}{c}{ BL } & \multicolumn{2}{c}{ H } \\
\hline & Seasonality & Fire & \multicolumn{2}{c}{ Seasonality } & Fire & Seasonality & Fire & Seasonality Fire \\
\hline HA & 0.52 & 0.45 & 0.11 & 0.06 & 0.14 & 0.02 & 0.21 & 0.38 \\
DHA & 0.40 & 0.25 & 0.29 & 0.32 & 0.23 & 0.25 & 0.35 & 1.58 \\
$\beta$-GLU & 0.17 & 0.09 & 0.05 & 0.24 & 0.34 & 0.04 & 0.44 & 0.67 \\
\hline
\end{tabular}

The CVs calculated for DHA highlighted the widest variability for seasonality in soils covered by $\mathrm{P}$ and for fire in soils covered by H (Table 4). The CVs for DHA were wider for seasonality than for fire only under P (Table 4); they were similar for seasonality and fire under $\mathrm{HO}$ and BL (Table 4); finally, they were wider for fire under $\mathrm{H}$ (Table 3).

The CVs calculated for $\beta$-GLU showed the widest variability both for seasonality and for fire in soils covered by $\mathrm{H}$ (Table 4); they were wider for seasonality than for fire in soils covered by P and BL (Table 4); conversely, they were wider for fire under HO (Table 4).

The results of the multiple linear regressions performed to highlight the main abiotic factors that controlled the enzymatic activities at different seasons and in UB and B soils are, respectively, reported in Tables 5 and 6 . In more detail, at different seasons, the factors that controlled the enzymatic activities regardless of the vegetation cover mainly were the available fraction of micro- and macro-nutrients, such as $\mathrm{Ca}, \mathrm{Fe}, \mathrm{Mn}, \mathrm{Mg}$, and $\mathrm{Na}$ (Table 5). Instead, in unburnt and burnt soils, the enzymatic activities were also controlled by the available fractions of metals such as $\mathrm{Al}, \mathrm{Cu}$, and $\mathrm{Pb}$ and by some other abiotic properties such as $\mathrm{WC}, \mathrm{pH}, \mathrm{OM}$, and total $\mathrm{C}$ and $\mathrm{N}$ (Table 6).

Table 5. Results of multiple linear regression analyses for soils under different vegetation covers and seasons. Significant results of multiple linear regression analyses of soil enzymatic activities (hydrolase: HA, dehydrogenase: DHA and $\beta$-glucosidase: $\beta$-GLU) in relationship with soil abiotic properties $(\mathrm{pH}$, water content: $\mathrm{WC}$, total concentration of carbon and nitrogen: $\mathrm{C}$ and $\mathrm{N}$, organic matter content: $\mathrm{OM}$, available fraction of $\mathrm{Al}, \mathrm{Ca}, \mathrm{Cu}, \mathrm{Fe}, \mathrm{K}, \mathrm{Mg}, \mathrm{Mn}, \mathrm{Na}$, and $\mathrm{Pb}$ ) in soils under different vegetation cover (P: pine; HO: holm oak; BL: black locust; H: herbs) at different seasons (S: summer 2018; F: fall 2018; W: winter 2019; Sp: spring 2019). Italics indicate the adjusted $\mathrm{R}^{2}$ of each model.

\begin{tabular}{cll}
\hline Vegetation Cover & Seasons & \multicolumn{1}{c}{ Multiple Regression Model } \\
\hline \multirow{2}{*}{ S } & $\mathrm{HA}=574.298-(2456.318 \times \mathrm{Mn})+(160.561 \times \mathrm{Fe})-(2.893 \times \mathrm{Mg})+(0.564 \times \mathrm{Ca}) R^{2}=0.62$ \\
& $\mathrm{DHA}=1.673+(0.962 \times \mathrm{Mn})-(0.115 \times \mathrm{Fe})-(0.00652 \times \mathrm{Mg})+(0.000201 \times \mathrm{Ca}) R^{2}=0.59$ \\
& $\mathrm{HA}=711.364-(0.0279 \times \mathrm{Mg}) R^{2}=0.58$ \\
& $\mathrm{DHA}=0.460+(0.000337 \times \mathrm{Mg}) R^{2}=0.70$ \\
& & $\beta-\mathrm{GLU}=406.772-(0.147 \times \mathrm{Mg}) R^{2}=0.72$ \\
& $\mathrm{HA}=528.337+(90.745 \times \mathrm{Mn})+(0.827 \times \mathrm{Fe})-(4.064 \times \mathrm{Mg})+(0.193 \times \mathrm{Ca}) R^{2}=0.80$ \\
& W & $\mathrm{DHA}=2.809-(0.0349 \times \mathrm{Mn})-(0.0291 \times \mathrm{Fe})+(0.0000110 \times \mathrm{Mg})-(0.000597 \times \mathrm{Ca}) R^{2}=0.78$ \\
& & $\beta-\mathrm{GLU}=-165.723+(84.836 \times \mathrm{Mn})+(1.887 \times \mathrm{Fe})-(0.892 \times \mathrm{Mg})+(0.129 \times \mathrm{Ca}) R^{2}=0.63$ \\
& $\mathrm{HA}=863.050+(109.434 \times \mathrm{Fe})-(82.788 \times \mathrm{Mg})+(0.302 \times \mathrm{Ca}) R^{2}=0.75$ \\
& $\mathrm{DHA}=-0.0664-(0.328 \times \mathrm{Mn})+(0.0971 \times \mathrm{Fe})-(0.0190 \times \mathrm{Mg})+(0.000516 \times \mathrm{Ca}) R^{2}=0.82$ \\
& $\beta-G L U=802.267+(16.795 \times \mathrm{Fe})-(24.072 \times \mathrm{Mg})+(0.0404 \times \mathrm{Ca}) R^{2}=0.84$
\end{tabular}


Table 5. Cont.

\begin{tabular}{|c|c|c|}
\hline Vegetation Cover & Seasons & Multiple Regression Model \\
\hline HO & $\begin{array}{c}\mathbf{F} \\
\mathbf{W}\end{array}$ & $\begin{array}{l}\text { HA }=622.891-(298.233 \times \mathrm{Mn})+(163.414 \times \mathrm{Fe})-(1.045 \times \mathrm{Mg})-(0.149 \times \mathrm{Ca}) R^{2}=0.81 \\
\mathrm{DHA}=2.674+(3.072 \times \mathrm{Mn})-(0.895 \times \mathrm{Fe})+(0.00618 \times \mathrm{Mg})+(0.000847 \times \mathrm{Ca}) R^{2}=0.72 \\
\beta-G L U=1113.367+(461.335 \times \mathrm{Mn})-(281.902 \times \mathrm{Fe})+(2.613 \times \mathrm{Mg})+(0.299 \times \mathrm{Ca}) R^{2}=0.84 \\
\beta-G L U=124.246+(17.697 \times \mathrm{Mn})-(0.846 \times \mathrm{Fe})+(0.0343 \times \mathrm{Mg})+(0.0251 \times \mathrm{Ca}) R^{2}=0.81 \\
\mathrm{HA}=1674.910-(7.305 \times \mathrm{Mn}) R^{2}=0.67 \\
\mathrm{DHA}=-0.429+(0.220 \times \mathrm{Mn}) R^{2}=0.64 \\
\beta-\mathrm{GLU}=363.752+(23.337 \times \mathrm{Mn}) R^{2}=0.63 \\
\mathrm{HA}=1181.300-(1.422 \times \mathrm{Mn})+(0.0471 \times \mathrm{Ca}) R^{2}=0.59 \\
\text { DHA }=-5.839+(0.285 \times \mathrm{Mn})+(0.000516 \times \mathrm{Ca}) R^{2}=0.60 \\
\beta-\mathrm{GLU}=-379.443+(32.196 \times \mathrm{Mn})+(0.0710 \times \mathrm{Ca}) R^{2}=0.77\end{array}$ \\
\hline BL & $\mathrm{Sp}$ & $\begin{array}{l}\mathrm{HA}=883.630-(3.860 \times \mathrm{Fe}) R^{2}=0.67 \\
\mathrm{DHA}=0.630+(0.136 \times \mathrm{Fe}) R^{2}=0.64 \\
\beta-\mathrm{GLU}=559.202-(17.491 \times \mathrm{Fe}) R^{2}=0.68 \\
\mathrm{HA}=-15233.898+(4.175 \times \mathrm{Mn})-(284.969 \times \mathrm{Fe})+(10.395 \times \mathrm{Mg})+(19.215 \times \mathrm{Na}) R^{2}=0.79 \\
\mathrm{DHA}=208.279-(0.680 \times \mathrm{Mn})+(3.825 \times \mathrm{Fe})-(0.105 \times \mathrm{Mg})-(0.261 \times \mathrm{Na}) R^{2}=0.83 \\
\beta-\mathrm{GLU}=7134.575-(34.826 \times \mathrm{Mn})+(130.277 \times \mathrm{Fe})-(2.506 \times \mathrm{Mg})-(8.946 \times \mathrm{Na}) R^{2}=0.88 \\
\mathrm{HA}=1399.255-(84.113 \times \mathrm{Mn})+(0.0368 \times \mathrm{Ca}) R^{2}=0.67 \\
\mathrm{DHA}=-0.0275-(0.179 \times \mathrm{Mn})-(0.0283 \times \mathrm{Fe})+(0.0110 \times \mathrm{Mg})+(0.000451 \times \mathrm{Ca}) R^{2}=0.82 \\
\mathrm{HA}=11274.414+(453.431 \times \mathrm{Mn})+(73.326 \times \mathrm{Fe})-(395.247 \times \mathrm{Mg})+(0.427 \times \mathrm{Ca}) R^{2}=0.73 \\
\text { DHA }=39.340+(2.090 \times \mathrm{Mn})-(0.537 \times \mathrm{Fe})-(1.436 \times \mathrm{Mg})+(0.00402 \times \mathrm{Ca}) R^{2}=0.84\end{array}$ \\
\hline $\mathbf{H}$ & $\mathrm{Sp}$ & $\begin{array}{l}\text { HA }=108.537+(919.366 \times \mathrm{Mn})+(0.229 \times \mathrm{Na}) R^{2}=0.65 \\
\text { DHA }=-3.254+(4.369 \times \mathrm{Mn})+(0.00622 \times \mathrm{Na}) R^{2}=0.67 \\
\beta-G L U=492.480-(120.333 \times \mathrm{Mn})-(0.643 \times \mathrm{Na} \\
\mathrm{HA}=-552.003-(498.522 \times \mathrm{Mn})-(12.495 \times \mathrm{Fe})+(4.447 \times \mathrm{Mg})+(0.522 \times \mathrm{Na}) R^{2}=0.62 \\
\text { DHA }=-31.416+(0.0372 \times \mathrm{Na}) R^{2}=0.89 \\
\beta-G L U=9.838-(194.965 \times \mathrm{Mn})-(8.247 \times \mathrm{Fe})+(2.389 \times \mathrm{Mg})-(0.0366 \times \mathrm{Na}) R^{2}=0.88 \\
\mathrm{HA}=-1819.093-(443.852 \times \mathrm{Mn})+(96.649 \times \mathrm{Fe})-(0.00256 \times \mathrm{Mg})+(1.839 \times \mathrm{Na}) R^{2}=0.79 \\
\mathrm{DHA}=-0.357+(0.548 \times \mathrm{Mn})+(0.0151 \times \mathrm{Fe})+(0.000637 \times \mathrm{Mg})-(0.000122 \times \mathrm{Na}) R^{2}=0.78 \\
\beta-\mathrm{GLU}=-2466.230+(0.290 \times \mathrm{Mg})+(2.824 \times \mathrm{Na}) R^{2}=0.67 \\
\mathrm{HA}=-3337.011+(2000.693 \times \mathrm{Mn})-(561.684 \times \mathrm{Fe})+(537.311 \times \mathrm{Mg})-(10.017 \times \mathrm{Na}) R^{2}=0.68 \\
\beta-\mathrm{GLU}=-685.425+(843.654 \times \mathrm{Mn})-(226.863 \times \mathrm{Fe})+(170.056 \times \mathrm{Mg})-(3.292 \times \mathrm{Na}) R^{2}=0.77\end{array}$ \\
\hline
\end{tabular}

Table 6. Results of multiple linear regression analyses for unburnt and burnt soils under different vegetation covers. Significant results of multiple linear regression analyses of soil enzymatic activities (hydrolase: HA, dehydrogenase: DHA and $\beta$-glucosidase: $\beta$-GLU) in relationship with soil abiotic properties ( $\mathrm{pH}$, water content: WC, total concentration of carbon and nitrogen: $\mathrm{C}$ and $\mathrm{N}$, organic matter content: $\mathrm{OM}$, available fraction of $\mathrm{Al}, \mathrm{Ca}, \mathrm{Cu}, \mathrm{Fe}, \mathrm{K}, \mathrm{Mg}, \mathrm{Mn}, \mathrm{Na}$, and $\mathrm{Pb}$ ) in unburnt (UB) and burnt (B) soils covered by different vegetation cover (P: pine; HO: holm oak; BL: black locust; H: herbs). Italics indicate the adjusted $R^{2}$ of each model.

\begin{tabular}{|c|c|c|}
\hline Vegetation Cover & Fire & Multiple Regression Model \\
\hline $\mathbf{P}$ & UB & $\begin{array}{l}\mathrm{HA}=1136.464-(232.733 \times \mathrm{Mn})+(19.395 \times \mathrm{Fe})-(1.311 \times \mathrm{Mg}) R^{2}=0.62 \\
\mathrm{DHA}=0.407+(0.980 \times \mathrm{Mn})-(0.126 \times \mathrm{Fe})+(0.00244 \times \mathrm{Mg}) R^{2}=0.58 \\
\beta-\mathrm{GLU}=-192.955+(263.969 \times \mathrm{Mn})-(17.263 \times \mathrm{Fe})+(0.816 \times \mathrm{Mg}) R^{2}=0.84 \\
\mathrm{HA}=-1647.699+(280.039 \times \mathrm{Cu})-(124.302 \times \mathrm{Pb})+(380.605 \times \mathrm{pH})-(115.994 \times \mathrm{C})-(767.327 \times \\
\mathrm{N})-(35.016 \times \mathrm{OM}) R^{2}=0.63 \\
\mathrm{DHA}=-3.280-(0.0869 \times \mathrm{Cu})+(0.0158 \times \mathrm{Pb})+(0.660 \times \mathrm{pH})-(0.0281 \times \mathrm{C})-(3.368 \times \mathrm{N})+ \\
(0.0288 \times \mathrm{OM}) R^{2}=0.73 \\
\beta-\mathrm{GLU}=123.032+(39.383 \times \mathrm{Cu})-(15.053 \times \mathrm{Pb})+(47.597 \times \mathrm{pH})-(14.792 \times \mathrm{C})-(510.130 \times \mathrm{N})- \\
(11.591 \times \mathrm{OM}) R^{2}=0.85\end{array}$ \\
\hline HO & UB & $\begin{array}{l}\mathrm{HA}=2276.187-(3.268 \times \mathrm{Mn})+(16.144 \times \mathrm{Fe})-(0.527 \times \mathrm{Mg})-(0.0698 \times \mathrm{Ca})-(2.135 \times \mathrm{Na})+ \\
(3.443 \times \mathrm{K})-(37.494 \times \mathrm{Al})+(1094.257 \times \mathrm{Cu})-(387.797 \times \mathrm{Pb})-(23.830 \times \mathrm{WC})-(26.092 \times \mathrm{pH}) R^{2} \\
=0.79 \\
\mathrm{DHA}=34.487+(0.298 \times \mathrm{Mn})+(0.0114 \times \mathrm{K})-(0.0277 \times \mathrm{Al})+(0.0130 \times \mathrm{WC})-(5.568 \times \mathrm{pH}) R^{2}= \\
0.71 \\
\beta-\mathrm{GLU}=1851.453+(18.433 \times \mathrm{Mn})+(20.033 \times \mathrm{Fe})+(0.171 \times \mathrm{Mg})-(0.00604 \times \mathrm{Ca})-(0.252 \times \mathrm{Na})+ \\
(0.167 \times \mathrm{K})-(21.172 \times \mathrm{Al})-(42.703 \times \mathrm{Cu})-(62.309 \times \mathrm{Pb})-(27.947 \times \mathrm{WC})-(152.040 \times \mathrm{pH}) R^{2} \\
=0.81\end{array}$ \\
\hline
\end{tabular}


Table 6. Cont.

\begin{tabular}{|c|c|c|}
\hline Vegetation Cover & Fire & Multiple Regression Model \\
\hline & B & $\begin{array}{l}\mathrm{HA}=5403.332-(54.698 \times \mathrm{Mn})+(60.722 \times \mathrm{Fe})-(3.431 \times \mathrm{Mg})+(0.224 \times \mathrm{Ca})-(4.760 \times \mathrm{Na})- \\
(2.999 \times \mathrm{K})-(41.834 \times \mathrm{Al}) R^{2}=0.69 \\
\mathrm{DHA}=12.864+(0.156 \times \mathrm{Mn})+(0.0182 \times \mathrm{Fe})-(0.00526 \times \mathrm{Mg})+(0.000709 \times \mathrm{Ca})-(0.0106 \times \mathrm{Na})- \\
(0.0106 \times \mathrm{K})-(0.0295 \times \mathrm{Al}) R^{2}=0.64 \\
\beta-\mathrm{GLU}=-877.077+(177.894 \times \mathrm{Mn})-(65.043 \times \mathrm{Fe})+(6.652 \times \mathrm{Mg})-(0.355 \times \mathrm{Ca})+(5.526 \times \mathrm{Na}) \\
-(4.029 \times \mathrm{K})-(1.771 \times \mathrm{Al}) R^{2}=0.71\end{array}$ \\
\hline BL & UB & $\begin{array}{l}\mathrm{HA}=1388.988+(2.103 \times \mathrm{Fe})-(1.760 \times \mathrm{K}) R^{2}=0.58 \\
\mathrm{DHA}=0.570+(0.0345 \times \mathrm{Fe})+(0.00250 \times \mathrm{K}) R^{2}=0.64 \\
\beta-\mathrm{GLU}=851.993+(0.0343 \times \mathrm{Fe})-(1.080 \times \mathrm{K}) R^{2}=0.71 \\
\mathrm{HA}=-19257.673-(187.103 \times \mathrm{Mn})+(48.790 \times \mathrm{Fe})-(2.244 \times \mathrm{Mg})+(0.234 \times \mathrm{Ca})-(2.767 \times \mathrm{Na})+ \\
(5.980 \times \mathrm{K})+(126.847 \times \mathrm{Al})+(717.584 \times \mathrm{Cu})-(323.015 \times \mathrm{Pb})+(70.248 \times \mathrm{WC})+(2462.431 \times \mathrm{pH}) \\
R^{2}=0.75\end{array}$ \\
\hline $\mathbf{H}$ & UB & $\begin{array}{l}\mathrm{HA}=1032.227-(0.109 \times \mathrm{Na})+(0.138 \times \mathrm{K})-(42.703 \times \mathrm{Al})-(158.552 \times \mathrm{Cu})+(2.663 \times \mathrm{Pb}) R^{2} \\
=0.81 \\
\mathrm{DHA}=1.559+(0.00207 \times \mathrm{Na})+(0.00273 \times \mathrm{K})-(0.274 \times \mathrm{Al})-(0.192 \times \mathrm{Cu})-(0.412 \times \mathrm{Pb}) R^{2}=0.73 \\
\beta-G L U=-116.330+(0.305 \times \mathrm{Na})+(0.0830 \times \mathrm{K})+(3.845 \times \mathrm{Al})-(4.984 \times \mathrm{Cu})+(18.675 \times \mathrm{Pb}) R^{2} \\
=0.87 \\
\mathrm{HA}=363.289+(65.104 \times \mathrm{Mn})-(10.143 \times \mathrm{Fe})+(0.171 \times \mathrm{Na}) R^{2}=0.75 \\
\mathrm{DHA}=0.488+(0.603 \times \mathrm{Mn})-(0.0359 \times \mathrm{Fe})-(0.000124 \times \mathrm{Na}) R^{2}=0.79 \\
\beta-\mathrm{GLU}=213.791+(187.946 \times \mathrm{Mn})+(12.950 \times \mathrm{Fe})-(0.277 \times \mathrm{Na}) R^{2}=0.73\end{array}$ \\
\hline
\end{tabular}

\section{Discussion}

In the Mediterranean environment, where the frequency of wildfires is high, it is useful to understand the impact of fires on soil microbial properties in order to prevent them and to put into practice strategies aiming to recover burnt areas. By now, it is known that fire effects on soils depend on fire intensity and frequency, but also on vegetation type, climate, fuel load, and topographic characteristics [37-39]; fire's effects on soil activities have been investigated to a lesser extent and the results of these studies are still controversial.

In the present research, the enzymatic activities mainly varied according to the vegetation covers, the seasons, and to a lesser extent according to the fire, as well as depending on the variations of soil abiotic properties [40,41]. In particular, in soils under the same vegetation cover the enzymatic activities were controlled by different soil properties according to seasons and fire occurrence, as shown by the multiple linear regressions. In fact, for all the vegetation covers, HA, DHA, and $\beta$-GLU activities over the seasons were controlled only by nutrient availabilities; whereas, in burnt soils they were also controlled by $\mathrm{pH}$, contents of water, and organic matter, $\mathrm{C}$ and $\mathrm{N}$, and by $\mathrm{Al}, \mathrm{Cu}$, and $\mathrm{Pb}$ availabilities. Seasonality exerted a strong effect on nutrient availabilities [42]; whereas, fire occurrence, besides its influence on nutrient and metal availabilities [14], also caused variations in soil pH, organic matter, and $\mathrm{C}$ and $\mathrm{N}$ contents [43]. These profound changes in soil abiotic properties, due to seasonality and fire effects, affected soil microbial and enzymatic activities [44].

However, some differences in properties affecting the enzymatic activities occurred among soils under the different investigated vegetation covers. More specifically, the availability of different macro- and micro-nutrients (i.e., $\mathrm{Ca}, \mathrm{Fe}, \mathrm{K}, \mathrm{Mg}$, $\mathrm{Mn}$, and $\mathrm{Na}$ ) regulated the temporal variation of each enzymatic activity in soils under different vegetation covers. This could be due to the heterogeneity of nutrient availability over the seasons under the same vegetation cover. For instance, $\mathrm{Mg}$ and $\mathrm{Na}$ significantly varied over the seasons in soils under all the investigated vegetation covers even if the highest values were frequently detected in fall. The high values of macronutrients found in fall could be due to the reduction in the plant uptake and nutrient requirements, and to the high litter accumulation that enhance the decomposition process [42]. Soils under pine showed differences in temporal variation of nutrient availability, as compared with the other vegetation covers, as the lowest values of Ca was detected in winter and those of $\mathrm{Mn}$ in summer, suggesting that, for this species, a temporal shift related to request of these elements occurred [45]. As observed for macro- and micro-nutrients, metals (i.e., $\mathrm{Al}, \mathrm{Cu}$, and $\mathrm{Pb}$ ) also significantly varied over the seasons in soils under all the investigated vegetation covers, with the exception of $\mathrm{Pb}$, 
which did not significantly vary under herbs. Despite of nutrients, metals showed similar temporal trends with the highest values in winter and the lowest in summer, suggesting that their mobility is more affected by microclimatic conditions rather than plants and uptake [32]. In addition, the pollution effect that is marked in winter when the anthropic activity is greater cannot be excluded [46].

The temporal variations in each enzymatic activity showed significant variations only for HA in soils under $\mathrm{HO}$ and $\mathrm{BL}$, and for DHA in soils under P. It is most likely that these variations for $\mathrm{HA}$ were due to $\mathrm{C}$ and $\mathrm{N}$ concentrations and to nutrient availability, especially $\mathrm{Ca}, \mathrm{K}$, and $\mathrm{Mg}$, and for DHA were due to $\mathrm{Ca}$ and $\mathrm{K}$ availability. Hydrolytic enzymes are a reliable index of soil bioactivity and fertility due to the association of humus and $\mathrm{C}$ and $\mathrm{N}$ contents [47]. Dehydrogenase intervenes in soil processes that create metabolic pathways for soil microorganisms, and it is also frequently used as a direct measure of soil microbial activity in relation to mineralization and the formation of humic substances [48]. It has been reported that the increase in dehydrogenase activity and microbial biomass were proportional to the addition of number and amount of nutrients [49]. However, the role of $\mathrm{pH}$ and water content in regulating the temporal variations of the enzymatic activities cannot be excluded, as they significantly varied for all the vegetation covers, with the exceptions of herbs. The highest values of HA activity in summer and winter under holm oak and for all the sampling seasons with the exception of spring under black locust could be due to the higher water and organic matter content. In fact, the catalytic efficiency of soil enzymes is highly affected by soil moisture content and $\mathrm{pH}[15,50,51]$.

The soil abiotic properties, with the exception of $\mathrm{Ca}$ and $\mathrm{Al}$ in soils under $\mathrm{HO}$, and of $\mathrm{pH}$ and $\mathrm{K}$ in soils under $\mathrm{H}$, as well as the enzymatic activities did not significantly change between unburnt and burnt soils within the same vegetation cover. Notwithstanding, an overall evaluation highlighted that fire differently affected soils under $\mathrm{HO}$ and $\mathrm{H}$ as compared with soils under $\mathrm{P}$ and $\mathrm{BL}$; in fact, in burnt soils under $\mathrm{HO}$ and $\mathrm{H}$, metal availability did not control the enzymatic activities, whereas the opposite occurred in those under P and BL. Fire occurrence induced transformations on soil metal content, exposing metals to the soil solution and enhancing their bioavailability [52]. The increased metal availability can cause the excessive accumulation of them in microorganisms, inducing oxidative stress or chelation of essential metabolites and causing enzyme inactivation and cell damage [52]. This was particularly evident in soils under pine, where the variations of several soil functions were of greater extent as compared with the other vegetation cover in Mediterranean area [14].

Fire, seasonality, and vegetation exerted a combined effect on soil abiotic properties and enzymatic activities [14,41]; in fact, fire and seasonality played opposing effects on soil abiotic properties within and among the vegetation covers that, on the whole, did not cause significant variations in soil enzymatic activities.

The narrowest heterogeneity of the enzymatic activities in responding to temporal and fire effects in soils under $\mathrm{HO}$ and BL suggested that these species confer a high resistance to soil variations. It is most likely that holm oak and black locust showing stability to perturbations contributed to maintaining homogeneous soil conditions that then ensured high microbial activity. This hypothesis is corroborated by previous studies for $\mathrm{HO}$ [41].

\section{Conclusions}

The results of the present research provided a contribution to the current knowledge about the impact of fire and seasonal variations on soil enzymatic activities in soils covered by different vegetation typical of Mediterranean area.

An overall evaluation highlighted that seasonality more than fire occurrence affected the soil enzymatic activities for all the investigated vegetation. Over the seasons, HA, DHA, and $\beta$-GLU activities in unburnt soils were controlled by nutrient availabilities; whereas, they were also affected by $\mathrm{pH}$, contents of water, organic matter, $\mathrm{C}$ and $\mathrm{N}$, and $\mathrm{Al}, \mathrm{Cu}$, and $\mathrm{Pb}$ availabilities. In burnt soils, a clear temporal trend appeared for metal availabilities that was higher in winter and lower in summer. 
Among the investigated vegetation, holm oak, showing the lowest coefficient of variation, conferred high stability to soils that were affected by both seasonality and fire.

Author Contributions: Conceptualization, V.M. and G.M.; methodology, V.M., L.S., G.S., P.M.; software, V.M., L.S.; validation, V.M., L.S., G.S., P.M., G.D.N., M.T., R.B., A.D.M. and G.M.; formal analysis, V.M., L.S., G.S., P.M., G.D.N., M.T.; investigation, V.M., L.S., A.D.M., G.M.; resources, G.M., L.S., V.M.; data curation, V.M., L.S., G.S., G.M.; writing-original draft preparation, V.M., L.S., G.M.; writing-review and editing, V.M., L.S., G.S., P.M., G.D.N., M.T., R.B., A.D.M. and G.M.; visualization, V.M., L.S., G.S., P.M., G.D.N., M.T., R.B., A.D.M. and G.M.; supervision, G.M.; project administration, G.M., R.B.; funding acquisition, G.M., R.B. All authors have read and agreed to the published version of the manuscript.

Funding: This research was funded by the collaboration of the Biology Department of University Federico II of Naples and the Vesuvius National Park within the "Azione di Sistema-Impatto antropico da pressione turistica nelle aree protette: interferenze su territorio e biodiversità" funded by "Ministero dell'Ambiente e della Tutela del Territorio e del Mare", Direttiva Conservazione della Biodiversità.

Institutional Review Board Statement: Not applicable.

Informed Consent Statement: Not applicable.

Data Availability Statement: The data presented in this study are available on request from the corresponding author. The data are not publicly available due to the privacy.

Conflicts of Interest: The authors declare no conflict of interest.

\section{References}

1. Karaca, A.; Cetin, S.C.; Turgay, O.C.; Kizilkaya, R. Soil enzymes as indication of soil quality. In Soil Enzymology; Shukla, G., Varma, A., Eds.; Springer: Berlin/Heidelberg, Germany, 2011; pp. 119-148.

2. Burns, R.G.; DeForest, J.L.; Marxsen, J.; Sinsabaugh, R.L.; Stromberger, M.E.; Wallenstein, M.D.; Weintraub, M.N.; Zoppini, A. Soil enzymes in a changing environment: Current knowledge and future directions. Soil Biol. Biochem. 2013, 58, 216-234. [CrossRef]

3. Kivlin, S.N.; Treseder, K.K. Soil extracellular enzyme activities correspond with abiotic factors more than fungal community composition. Biogeochemistry 2014, 117, 23-37. [CrossRef]

4. Henry, H.A. Soil extracellular enzyme dynamics in a changing climate. Soil Biol. Biochem. 2012, 47, 53-59. [CrossRef]

5. Cao, R.; Yang, W.; Chang, C.; Wang, Z.; Wang, Q.; Li, H.; Tan, B. Differential seasonal changes in soil enzyme activity along an altitudinal gradient in an alpine-gorge region. Appl. Soil Ecol. 2021, 166, 104078. [CrossRef]

6. Kivlin, S.N.; Hawkes, C.V. Spatial and temporal turnover of soil microbial communities is not linked to function in a primary tropical forest. Ecology 2020, 101, e02985. [CrossRef]

7. Midgley, M.G.; Phillips, R.P. Spatio-temporal heterogeneity in extracellular enzyme activities tracks variation in saprotrophic fungal biomass in a temperate hardwood forest. Soil Biol. Biochem. 2019, 138, 107600. [CrossRef]

8. Li, Z.; Yang, W.; Yue, K.; Justine, M.F.; He, R.; Yang, K.; Zhuang, L.; Wu, F.; Tan, B.; Zhang, L.; et al. Effects of snow absence on winter soil nitrogen dynamics in a subalpine spruce forest of southwestern China. Geoderma 2017, 307, 107-113. [CrossRef]

9. Akinyemi, D.S.; Zhu, Y.; Zhao, M.; Zhang, P.; Shen, H.; Fang, J. Response of soil extracellular enzyme activity to experimental precipitation in a shrub-encroached grassland in Inner Mongolia. Glob. Ecol. Conserv. 2020, 23, e01175. [CrossRef]

10. Ouyang, Y.; Li, X. Effect of repeated drying-rewetting cycles on soil extracellular enzyme activities and microbial community composition in arid and semi-arid ecosystems. Eur. J. Soil Biol. 2020, 98, 103187. [CrossRef]

11. Loeppmann, S.; Blagodatskaya, E.; Pausch, J.; Kuzyakov, Y. Substrate quality affects kinetics and catalytic efficiency of exoenzymes in rhizosphere and detritusphere. Soil Biol. Biochem. 2016, 92, 111-118. [CrossRef]

12. Xu, Z.; Zhang, T.; Wang, S.; Wang, Z. Soil $\mathrm{pH}$ and $\mathrm{C} / \mathrm{N}$ ratio determines spatial variations in soil microbial communities and enzymatic activities of the agricultural ecosystems in Northeast China: Jilin Province case. Appl. Soil Ecol. $2020,155,103629$. [CrossRef]

13. Santorufo, L.; Cortet, J.; Nahmani, J.; Pernin, C.; Salmon, S.; Pernot, A.; Morel, J.L.; Maisto, G. Responses of functional and taxonomic collembolan community structure to site management in Mediterranean urban and surrounding areas. Eur. J. Soil Biol. 2015, 70, 46-57. [CrossRef]

14. Santorufo, L.; Memoli, V.; Panico, S.C.; Santini, G.; Barile, R.; Di Natale, G.; Trifuoggi, M.; De Marco, A.; Maisto, G. Early post-fire changes in properties of Andosols within a Mediterranean area. Geoderma 2021, 394, 115016. [CrossRef]

15. Memoli, V.; Eymar, E.; García-Delgado, C.; Esposito, F.; Panico, S.C.; De Marco, A.; Barile, R.; Maisto, G. Soil element fractions affect phytotoxicity, microbial biomass and activity in volcanic areas. Sci. Total Environ. 2018, 636, 1099-1108. [CrossRef] [PubMed]

16. Memoli, V.; Panico, S.C.; Santorufo, L.; Barile, R.; Di Natale, G.; Di Nunzio, A.; Toscanesi, M.; Trifuoggi, M.; De Marco, A.; Maisto, G. Do wildfires cause changes in soil quality in short term? Int. J. Environ. Res. Public Health 2020, 17, 5343. [CrossRef] [PubMed] 
17. Saá, A.; Trasar-Cepeda, M.C.; Gil-Sotres, F.; Carballas, T. Changes in soil phosphorus and acid phosphatase activity immediately following forest fires. Soil Biol. Biochem. 1993, 25, 1223-1230. [CrossRef]

18. Boerner, R.E.J.; Giai, C.; Huang, J.; Miesel, J.R. Initial effects of fire and mechanical thinning on soil enzyme activity and nitrogen transformations in eight North American forest ecosystems. Soil Biol. Biochem. 2008, 40, 3076-3085. [CrossRef]

19. Rutigliano, F.A.; D'ascoli, R.; Virzo De Santo, A. Soil microbial metabolism and nutrient status in a Mediterranean area as affected by plant cover. Soil Biol. Biochem. 2004, 436, 1719-1729. [CrossRef]

20. Sardans, J.; Peñuelas, J. Drought decreases soil enzyme activity in a Mediterranean Quercus ilex L. forest. Soil Biol. Biochem. 2005, 37, 455-461. [CrossRef]

21. Guénon, R.; Gros, R. Frequent-wildfires with shortened time-since-fire affect soil microbial functional stability to drying and rewetting events. Soil Biol. Biochem 2013, 57, 663-674. [CrossRef]

22. Bárcenas-Moreno, G.; García-Orenes, F.; Mataix-Beneyto, J.; Bååth, E. Plant species influence on soil microbial short-term response after fire simulation. Plant Soil 2014, 374, 701-713. [CrossRef]

23. Stott, D.E.; Andrews, S.S.; Liebig, M.A.; Wienhold, B.J.; Karlen, D.L. Evaluation of $\beta$-glucosidase activity as a soil quality indicator for the soil management assessment framework. Soil Sci. Soc. Am. J. 2010, 74, 107-119. [CrossRef]

24. Dotaniya, M.L.; Aparna, K.; Dotaniya, C.K.; Singh, M.; Regar, K.L. Role of Soil Enzymes in Sustainable Crop Production. Enzym. Food Biotechnol. Prod. Appl. Future Prospect. 2019, 569-589. [CrossRef]

25. Adam, G.; Duncan, H. Development of a sensitive and rapid method for the measurement of total microbial activity using Fluorescein Diacetate (FDA) in a range of soils. Soil Biol. Biochem. 2001, 33, 943-951. [CrossRef]

26. Huang, W.; Xu, Z.; Chen, C.; Zhou, G.; Liu, J.; Abdullah, K.M.; Reverchon, F.; Liu, X. Short-term effects of prescribed burning on phosphorus availability in a suburban native forest of subtropical Australia. J. Soils Sediments 2013, 13, 869-876. [CrossRef]

27. Holden, S.R.; Gutierrez, A.; Treseder, K.K. Changes in soil fungal communities, extracellular enzyme activities, and litter decomposition across a fire chronosequence in Alaskan boreal forests. Ecosystems 2013, 16, 34-46. [CrossRef]

28. Wolińska, A.; Stepniewska, Z. Dehydrogenase activity in the soil environment. Dehydrogenases 2012, 10, $183-210$.

29. Henriksson, G.; Sild, V.; Szabó, I.J.; Pettersson, G.; Johansson, G. Substrate specificity of cellobiose dehydrogenase from Phanerochaete chrysosporium. Biochim. Biophys. Acta Protein Struct. Mol. Enzymol. 1998, 1383, 48-54. [CrossRef]

30. Vega, J.A.; Fontùrbel, T.; Merino, A.; Fernàndez, C.; Ferreiro, A.; Jiménez, E. Testing the suitability of visual indicators of soil burn severity to reflect changes in soil chemical and microbial properties in pine stands and shrublands. Plant Soil 2013, 369, 73-91. [CrossRef]

31. Saulino, L.; Rita, A.; Migliozzi, A.; Maffei, C.; Allevato, E.; Garonna, A.P.; Saracino, A. Detecting burn severity across Mediterranean forest types by coupling medium-spatial resolution satellite imagery and field data. Remote Sens. 2020, 12, 741. [CrossRef]

32. Panico, S.C.; Memoli, V.; Santorufo, L.; Esposito, F.; De Marco, A.; Barile, R.; Maisto, G. Linkage between site features and soil characteristics within a Mediterranean volcanic area. Front. For. Glob. Chang. 2021, 3, 621231. [CrossRef]

33. Lindsay, W.N.; Norwell, W.A. Development of a DTPA micronutrient soil test. Agron. Abstr. 1969, 84, $69-87$.

34. Tabatabai, A. Soil Enzymes. In Methods of Soil Analysis; Page, A.L., Miller, R.H., Keeney, D.R., Eds.; E-Publishing Inc.: Madison, WI, USA, 1982; pp. 903-947.

35. Tabatabai, M.A.; Bremner, J.M. Use of p-nitrophenyl phosphate for assay of soil phosphatase activity. Soil Biol. Biochem. 1969, 1, 301-307. [CrossRef]

36. Oksanen, J.; Blanchet, G.; Roeland, K.; Legendre, P.; Minchin, P.R.; O’Hara, R.B.; Simpson, G.L.; Solymos, P.; Stevens, M.H.H.; Wagner, H. Vegan: Community Ecology Package. R Package 2014, 280.

37. Certini, G. Effects of fire on properties of forest soils: A review. Oecologia 2005, 143, 1-10. [CrossRef] [PubMed]

38. Brando, P.M.; Oliveria-Santos, C.; Rocha, W.; Cury, R.; Coe, M.T. Effects of experimental fuel additions on fire intensity and severity: Unexpected carbon resilience of a neotropical forest. Glob. Chang. Biol. 2016, 2516-2525. [CrossRef] [PubMed]

39. Panico, S.C.; Ceccherini, M.T.; Memoli, V.; Maisto, G.; Pietramellara, G.; Barile, R.; De Marco, A. Effects of different vegetation types on burnt soil properties and microbial communities. Int. J. Wildland Fire 2020, 29, 628-636. [CrossRef]

40. Moya, D.; Madrigal, J.; Fontúrbel, T.; Marino, E.; Hernando, C.; Guijarro, M.; Fernández, C.; Jiménez, E.; Vega, J.A.; de las Heras, J. Fire severity assessments in both the laboratory and the field. In Fire Effects on Soil Properties; CRC Press: Boca Raton, FL, USA; Taylor \& Francis Group: Oxfordshire, UK, 2018; pp. 241-265.

41. Memoli, V.; Panico, S.C.; Barile, R.; Di Natale, G.; Trifuoggi, M.; De Marco, A.; Maisto, G. Stability of mediterranean burnt soils under different plant covers. Catena 2021, 206, 105581. [CrossRef]

42. Omer, M.; Idowu, O.J.; Ulery, A.L.; VanLeeuwen, D.; Guldan, S.J. Seasonal changes of soil quality indicators in selected arid cropping systems. Agriculture 2018, 8, 124. [CrossRef]

43. Zavala, L.; de Celis, R.; Jordán, A. How wildfires affect soil properties. A brief review. Cuad. Investig. Geográfica/Geogr. Res. Lett. 2014, 40, 311-332. [CrossRef]

44. Lopez-Póma, R.; Bautista, S. Plant regeneration functional groups modulate the response to fire of soil enzyme activities in a Mediterranean shrubland. Soil Biol. Biochem. 2014, 79, 5-13. [CrossRef]

45. Helmisaari, H. Nutrient retranslocation within the foliage of Pinus sylvestris. Tree Physiol. 1990, 10, 45-58. [CrossRef]

46. Memoli, V.; Esposito, F.; Panico, S.C.; De Marco, A.; Barile, R.; Maisto, G. Evaluation of tourism impact on soil metal accumulation through single and integrated indices. Sci. Total Environ. 2019, 682, 685-691. [CrossRef] [PubMed] 
47. Baležentiené, L. Hydrolases related to $C$ and $N$ cycles and soil fertility amendment: Responses to different management styles and agro-ecosystems. Pol. J. Environ. Stud. 2012, 21, 1153-1159.

48. Bueis, T.; Turrión, M.B.; Bravo, F.; Pando, V.; Muscolo, A. Factors determining enzyme activities in soils under Pinus halepensis and Pinus sylvestris plantations in Spain: A basis for establishing sustainable forest management strategies. Ann. For. Sci. 2018, 75, 34. [CrossRef]

49. Adak, T.; Singh, A.; Kumar, K.; Shukla, S.K.; Singh, A.; Kumar Singh, V. Soil organic carbon, dehydrogenase activity, nutrient availability and leaf nutrient content as affected by organic and inorganic source of nutrient in mango orchard soil. J. Soil Sci. Plant. Nutr. 2014, 2, 394-406. [CrossRef]

50. Zhao, J.; Dong, Y.; Xie, X.; Li, X.; Zhang, X.; Shen, X. Effect of annual variation in soil pH on available soil nutrients in pear orchards. Acta Ecol. Sin. 2011, 31, 212-216. [CrossRef]

51. Zhang, W.; Calvo-Polanco, M.; Chen, Z.C.; Zwiazek, J.J. Growth and physiological responses of trembling aspen (Populus tremuloides), white spruce (Picea glauca) and tamarack (Larix laricina) seedlings to root zone pH. Plant Soil 2013, 373, 775-786. [CrossRef]

52. Terzano, R.; Rascio, I.; Allegretta, I.; Porfido, C.; Spagnuolo, M.; Khanghahi, M.Y.; Crecchio, C.; Sakellariadou, F.; Gattullo, C.E. Fire effects on the distribution and bioavailability of potentially toxic elements (PTEs) in agricultural soils. Chemosphere 2021, 281, 130752. [CrossRef] [PubMed] 